\title{
MULTISCALE COUPLINGS IN PROTOTYPE HYBRID DETERMINISTIC/STOCHASTIC SYSTEMS: PART I, DETERMINISTIC CLOSURES*
}

\author{
M.A. KATSOULAKIS ${ }^{\dagger}$, A.J. MAJDA ${ }^{\ddagger}$, AND A. SOPASAKIS ${ }^{\S}$
}

\begin{abstract}
We introduce and study a class of model prototype hybrid systems comprised of a microscopic stochastic surface process modeling adsorption/desorption and/or surface diffusion of particles coupled to an ordinary differential equation (ODE) displaying bifurcations excited by a critical noise parameter. The models proposed here are caricatures of realistic systems arising in diverse applications ranging from surface processes and catalysis to atmospheric and oceanic models.

We obtain deterministic mesoscopic models from the hybrid system by employing two methods: stochastic averaging principle and mean field closures. In this paper we focus on the case where phase transitions do not occur in the stochastic system. In the averaging principle case a faster stochastic mechanism is assumed compared to the ODE relaxation and a local equilibrium is induced with respect to the Gibbs measure on the lattice system. Under these circumstances remarkable agreement is observed between the hybrid system and the averaged system predictions. We exhibit several Monte Carlo simulations testing a variety of parameter regimes and displaying numerically the extent, limitations and validity of the theory. As expected fluctuation driven rare events do occur in several parameter regimes which could not possibly be captured by the deterministic averaging principle equation.
\end{abstract}

\section{Introduction}

Couplings of atomistic or molecular, and more generally microscopic stochastic models to deterministic macroscopic ordinary and partial differential equations (ODE/PDE) are commonplace in a wide array of applications, ranging from catalysis and deposition processes to stochastic models for tropical and open ocean convection and complex biological networks, see for instance [18, 24]. In this category of problems microscopic stochastic processes, typically simulated by Monte Carlo (MC) methods, model small scale activity on an interface or boundary layer, e.g. adsorption, desorption, surface reaction and surface diffusion of particles, in contact with a gas/fluid phase. The micromechanisms are interrelated with the large scale adjacent fluid flow modeled by continuum ODE/PDE describing the evolution of fluid and thermodynamic variables.

The overarching challenge in all these hybrid problems is essentially two-fold: a first difficulty arises in the direct numerical simulation of realistic size systems due to scale disparities between the discrete stochastic microscopic models and the continuum macroscopic equations; secondly, the fact that the coupled systems have nonlinear interactions across a wide range of scales, implies that the stochasticity inherited from the microscopic model can play a subtle but important role in the dynamic behavior of the overall system. In this paper we address directly or indirectly both issues in the context of proposed prototype mathematical models of a deterministic ODE coupled with a stochastic spin flip/spin exchange Ising model, that capture essential features of complex hybrid systems. More specifically the models put forward here, (a) allow E.

*Received: April 4, 2004; accepted (in revised version): May 18, 2004. Communicated by Weinan

${ }^{\dagger}$ Department of Mathematics and Statistics, University of Massachusetts, Amherst, MA 010039305, USA.

${ }^{\ddagger}$ Courant Institute of Mathematical Sciences and Center for Atmospheric and Ocean Sciences, New York University, New York, NY 10012, USA.

$\S$ Department of Mathematics and Statistics, University of Massachusetts, Amherst, MA 010039305, USA. 
for testing the derivation of computationally inexpensive mesoscopic deterministic and stochastic models for the average behavior of the hybrid systems in various asymptotic limits, and (b) render computationally feasible detailed comparisons of the derived mesoscopic theories against direct numerical simulations of the full hybrid system; although remarkable agreement is observed, long-time simulations and asymptotic analysis in a linearized stochastic PDE limit strongly suggest that fluctuation-driven rare events do occur in several parameter regimes and are not captured by the deterministic mesoscopic equations. Furthermore fluctuations play a dominant role in regimes where phase transitions occur in the stochastic system. As a result it is clear that we need to develop mathematical strategies that allow fluctuations to be properly and systematically included in the deterministic mesoscopic models. In a companion publication [8] we address this issue by employing recently developed coarse-grained stochastic models $[9,10,11]$, in order to describe the microscopic mechanisms with a hierarchy of coarser (and thus computationally preferable) but still discrete, stochastic observables. In this spirit, hybrid coarse-grained models were already introduced in [13] as stochastic parameterizations of unresolved features of tropical convection; such models provide a computationally efficient stochastic model whose predictions can be directly related to observational data. In the next paragraphs we motivate the proposed prototype systems by first discussing two concrete applications that have partly inspired the choice of the models and the ensuing analysis and simulations discussed in this paper.

Microscopic descriptions of surface mechanisms on catalytic reactors, [25] involve adsorption, desorption, reaction and surface diffusion processes and can be modeled by stochastic Ising systems with spin flip and spin exchange dynamics, [17]. On the other hand, the gas-phase modeling is based on continuum PDE and includes equations for transport, thermodynamics and gas-phase reactions, [2]. The microscopic stochastic surface processes are coupled to the continuum PDE via boundary conditions and adsorption/desorption rates to and from the surface. One of the first examples of hybrid systems studied in the literature can be found in [24]. This physical system was first studied in the mean-field (MF) regime in [12], and includes an ODE describing the spatially uniform coverage on the catalytic surface. The resulting system of two coupled ODEs predicts oscillations both in the coverage and the pressure but it disregards fluctuations, detailed interactions and spatial inhomogeneities. Comparisons between MF and MC simulations [24] show that in many parameter regimes there is substantial agreement attributed to the coupling with the well-mixed gas-phase; drastic discrepancies are also observed, as well as a variety of dynamic and equilibrium behaviors, depending on the separation of time scales between individual micromechanisms and the time scale of the ODE, and also the presence of phase transitions for the surface process.

In the same broad mathematical context of phenomena with fluid-surface couplings, stochastic models for unresolved features in tropical convection were recently proposed in [18]. Due to the significant impact of tropical convection to the shortterm climate, it is crucial to be incorporated to general circulation models (GCM), for accurate short-term weather/climate prediction. Currently, the smallest mesh sizes for the discretization of the fluid and thermodynamic equations in GCM range from $50-300 \mathrm{~km}$. However deep penetrative convection towers are of the order of $1-10 \mathrm{~km}$ in the horizontal variable and need to be incorporated as a subgrid scale model. The process of modeling these unresolved features is referred to as "parametrization" and is carried out according to various, usually deterministic, recipes [6]. Related issues 
also arise in numerous Atmosphere/Ocean problems such as the modeling of sea-ice cover, open ocean convection and deep convective clouds in the tropics. In spite of the progress in deterministic parameterizations, many significant observed features of tropical convection cannot still be reproduced with current GCM [6]. In this direction, a new stochastic parametrization for tropical convection was introduced in [18] which is roughly analogous to the hybrid catalysis model described above. The significance of this stochastic/deterministic coupling is underscored by the sensitivity of the PDE systems on the (area fraction) parameter $\bar{\sigma}$. It was shown in [19] that in models with two vertical baroclinic modes (and is known to occur in single vertical baroclinic mode models), waves change from a stable convectively damped regime to a scale-selective unstable convective regime when $\bar{\sigma}$ is varied in a suitable interval.

As it is evident from the two examples discussed above, hybrid systems are expected to have a host of complicated and nonlinear interactions between different scales, fluctuations and parameter regimes. A common feature of this class of hybrid models is that the influence of noise can be critical since in various parameter regimes they can exhibit average behavior characterized by oscillations, Hopf and saddle node bifurcations, heteroclinic orbits and possibly chaos. It is therefore clear that we need to develop prototype problems that can capture such essential features of the physical models but which still are amenable to asymptotics, analysis and tractable computations. This in turn will allow us to benchmark new modeling and computational ideas, such as coarse-graining [9, 10, 11], stochastic averaging [7], and mode elimination [20]. More specifically we introduce in Section 2 a system of microscopic stochastic surface processes modeling adsorption/desorption and/or surface diffusion of particles with spin flip/exchange Ising systems, coupled to an ODE that serves as a caricature of the gas-phase, exhibiting a similar variety of dynamic and stability behaviors in different parameter regimes. Examples of such ODEs considered here are scalar equations exhibiting bi-stability and saddle-node bifurcations, as well as a spatially homogeneous complex Ginzburg-Landau equation exhibiting a Hopf bifurcation behavior.

Our work on these problems addresses two main themes. First in Section 3, we derive deterministic mesoscopic models from the hybrid system by employing a stochastic averaging principle to the microscopic lattice model (see [7], [20] for stochastic averaging for SDE). These derivations are carried out rigorously when the stochastic mechanism is faster than the gas-phase (ODE) relaxation, inducing a local equilibrium with respect to the Gibbs measure on the lattice system. We also address briefly the implications of the possibility of phase transitions in the spin flip model, although in this paper we focus on the much simpler case where phase transitions do not occur. Other mesoscopic, spatially distributed equations can be derived without assuming time-scale separation but rather long range interactions on the microscopic processes. In both asymptotic regimes the resulting averaged equations can be thought of as deterministic coarse-grained models providing some first intuition on the interplay of various mechanisms and models.

In Section 4 we carry out extensive comparative simulations with both the hybrid system and the mesoscopic deterministic equation derived through stochastic averaging and observe remarkable agreement. However simulations for very long times with our hybrid prototype models, as well as "crude" linearization arguments, strongly suggest that fluctuation-driven rare events do occur in several parameter regimes and are not captured by the deterministic averaged equations discussed above. Furthermore, random fluctuations are especially important in phase transition regimes. As a result it is clear that fluctuations need to be properly included in any coarse-grained 
mesoscopic model. In a follow-up publication [8] we address this issue by employing recently developed coarse-grained stochastic models $[9,10,11]$, in order to describe the microscopic mechanisms with a hierarchy of coarser stochastic observables.

\section{A mathematical prototype hybrid model}

We now introduce the microscopic spin flip stochastic Ising process $\left\{\sigma_{t}\right\}_{t \geq 0}$, modeling the adsorption and desorption of particles on a one-dimensional surface, coupled to an ODE that serves as a caricature of an overlying gas-phase dynamics. The stochastic process is completely determined by its generator $L$ and the coupled system is written as

$$
\begin{aligned}
\frac{d}{d t} E f(\sigma) & =E L f(\sigma) \\
\frac{d}{d t} \vec{X} & =f(\vec{X}, \bar{\sigma})
\end{aligned}
$$

for which we provide all the details below. Examples of ODEs such as (2.2) considered here are scalar equations with bistable behavior or saddle node bifurcations, as well as a spatially homogeneous complex Ginzburg-Landau equation exhibiting Hopf bifurcations. In addition to the spin flip mechanism, we can also consider spin exchange, as well as combined mechanisms. In this paper we concentrate only on one of them, namely spin flips.

2.1. Microscopic Arrhenius spin-flip / spin-exchange dynamics. We consider a microscopic stochastic model defined on a periodic lattice of size $N$ which we denote by $\mathcal{L}=\{1,2, \ldots, N\}$. At each lattice site $x \in \mathcal{L}$, an order parameter $\sigma$, is allowed to take the values 0 or 1 . In accordance with the classical Ising model we refer to the order parameter as spin. We will assume that sites cannot be occupied by more than one particle (exclusion principle). A spin configuration $\sigma$ is an element of the configuration space $\Sigma=\{0,1\}^{\mathcal{L}}$ and we write $\sigma=\{\sigma(x): x \in \mathcal{L}\}$ denoting by $\sigma(x)$ the spin at $x$. Physically this mechanism may describe the desorption of a particle from a surface described by the lattice to the gas phase above and conversely the absorption of a particle from the gas phase to the surface. Similarly it can describe phase transitions without order parameter conservation.

The stochastic process $\left\{\sigma_{t}\right\}_{t \geq 0}$ is a continuous time jump Markov process on $L^{\infty}(\Sigma, R)$ with generator, [14],

$$
L_{a d} f(\sigma)=\sum_{x \in \mathcal{L}} c(x, \sigma)\left[f\left(\sigma^{x}\right)-f(\sigma)\right]
$$

for any test function $f \in L^{\infty}(\Sigma, R)$. Here $c(x, \sigma)$ denotes the rate of the process (see $2.6)$ and $\sigma^{x}$ signifies the configuration after a flip at $x$,

$$
\sigma^{x}(y)=\left\{\begin{array}{cc}
1-\sigma(x), & \text { if } y=x \\
\sigma(y), & \text { if } y \neq x
\end{array}\right.
$$

Since there are $N=|\mathcal{L}|$ sites on the lattice then the system can be in any of $2^{|\mathcal{L}|}$ possible states while the energy of any particular state is given by the following Hamiltonian,

$$
H(\sigma)=-\frac{1}{2} \sum_{x \in \mathcal{L}} \sum_{y \neq x} J(x, y) \sigma(x) \sigma(y)+\sum_{x} h \sigma(x),
$$


where $J(x, y)$ is an interparticle potential and $h$ is a given external potential. We let,

$$
J(x, y)=\gamma J(\gamma|x-y|)
$$

where $\gamma$ is a parameter prescribing the range of microscopic interactions. Here $J$ is assumed to be even, $J(r)=J(-r)$ and as an example, can take a form similar to [9],

$$
J(r)= \begin{cases}J_{0} & \text { if } 0 \leq r \leq 1 \\ 0 & \text { otherwise }\end{cases}
$$

where $J_{0}$ is a parameter which based on its sign describes attractive, repulsive or nointeractions. For attractive microscopic interactions for instance $J_{0}$ is positive. We let $\gamma=1 /(2 L+1)$ where $L$ denotes the interaction radius.

Applying a spin flip stochastic model we create new states from old ones generated by a Markov process as described below. Equilibrium states of the stochastic model are described by the Gibbs states at the prescribed temperature $T$. If we denote the inverse temperature by $\beta=1 /(k T)$ we then have,

$$
\mu_{\beta, N}(d \sigma)=\frac{1}{Z} e^{-\beta H(\sigma)} P_{N}(d \sigma)
$$

where $P_{N}(d \sigma)$ denotes the (product) prior distribution on $\mathcal{L}$,

$$
P_{N}(d \sigma)=\prod_{x \in \mathcal{L}} \rho(d \sigma(x)) \text { and } \rho(\sigma(x)=0)=\frac{1}{2}, \quad \rho(\sigma(x)=1)=\frac{1}{2} .
$$

Here $Z$ is the partition function, guaranteeing that (2.5) is a probability measure. Note that $k=1.38 \times 10^{-23} \mathrm{JK}^{-1}$ is the Boltzmann constant. As is common we measure temperature in energy units and therefore we take $k=1$.

In this model we implement spin flip Arrhenius dynamics. Therefore under this type of mechanism the simulation is driven based on the energy barrier a particle has to overcome in flipping from one state to another. For Metropolis and other choices of dynamics see [9]. The Arrhenius spin flip rate $c(x, \sigma)$ at lattice site $x$ and spin configuration $\sigma$ is given by

$$
c(x, \sigma)= \begin{cases}c_{d} e^{-\beta\left[U_{0}+U(x)\right]}, & \text { when } \sigma(x)=0 \\ c_{a} & \text { when } \sigma(x)=1 .\end{cases}
$$

where,

$$
U(x)=\sum_{\substack{z \neq x \\ z \in \mathcal{L}}} J(x, z) \sigma(z)-h .
$$

with absorption/desorption constants,

$$
c_{a}=c_{d}=1 / \tau_{I} .
$$

and $\tau_{I}$ denotes the characteristic time of the stochastic process. Here $U_{0}$ represents the energy associated with the surface binding of the particle at location $x$ which we set to $U_{0}=0$. The ODE (2.2) and the stochastic system (2.1) are coupled via, respectively, the external field,

$$
h \equiv h(\vec{X})
$$


and the area fraction (or total coverage) defined as the spatial average of the stochastic process $\sigma$,

$$
\bar{\sigma}=\frac{1}{N} \sum_{x \in \mathcal{L}} \sigma(x)
$$

In general we write the spin flip rate (2.6) as,

$$
c(x, \sigma):=c_{a}(x, \sigma)+c_{d}(x, \sigma)=c_{a}(1-\sigma(x))+c_{d} \sigma(x) \exp \left(-\beta\left(U_{0}+U(x)\right)\right.
$$

and therefore the probability of a spin flip at $x$ during time $[t, t+\Delta t]$ is,

$$
c(x, \sigma) \Delta t+O\left(\Delta t^{2}\right)
$$

The dynamics as described here leave the Gibbs measure (2.5) invariant, since they satisfy the detailed balance condition

$$
c(x, \sigma)=c\left(x, \sigma^{x}\right) \exp \left(-\beta \Delta_{x} H(\sigma)\right)
$$

where

$$
\Delta_{x} H(\sigma)=H\left(\sigma^{x}\right)-H(\sigma) .
$$

In the case of a surface diffusion process we implement spin-exchange Arrhenius dynamics. A spin exchange between the neighboring sites $x$ and $y$ is a spontaneous exchange of the values of the order parameter $x$ and $y$. Physically this mechanism describes the diffusion of a particle on a flat surface. Note that sites cannot be occupied by more than one particle (exclusion principle). As in the spin flip dynamics, a spin exchange (for nearest neighbors $x$ and $y$ ) occurs with rate $c(x, y, \sigma)$ satisfying the detailed balance law

$$
c(x, y, \sigma)= \begin{cases}c_{\text {diff }} e^{-\beta\left[U_{0}+U(x)\right]} & \text { when } \sigma(x)=1, \sigma(y)=0, \\ c_{\text {diff }} e^{-\beta\left[U_{0}+U(y)\right]} & \text { when } \sigma(x)=0, \sigma(y)=1, \\ 0 & \text { otherwise }\end{cases}
$$

where $c_{\text {diff }}=1 / \tau_{\text {diff }}$. The corresponding generator for this process, $L_{\text {diff } f}$, satisfies the equivalent of (2.3) with rate (2.12). As a result the corresponding generator, $L$, in (2.1) of the combined mechanism for the dynamics comprised of spin flip and spin-exchange is given via,

$$
L=L_{\text {diff }}+L_{a d}
$$

Similar combined mechanisms were considered in [24]. In this paper we focus for simplicity on the spin flip case only.

2.2. Couplings with deterministic systems. One of the types of ODE (2.2) we consider is the well-known $[1,16]$, spatially homogeneous cubic Complex Ginzburg Landau equation,

$$
\tau_{c} \frac{d \vec{X}}{d t}=(a(\bar{\sigma})+i \omega) \vec{X}-\tilde{\gamma}|\vec{X}|^{2} \vec{X}+\gamma \vec{X}^{*}
$$

where $\vec{X}=X+i Y$ and $\vec{X}^{*}=X-i Y$. In matrix form (2.13) is written as

$$
\frac{d \vec{X}}{d t}=f(\vec{X}, \bar{\sigma})=\frac{1}{\tau_{c}}\left[A \vec{X}-\tilde{\gamma}|\vec{X}|^{2} \vec{X}\right], \text { for } A=\left(\begin{array}{cc}
a(\bar{\sigma})+\gamma & -\omega \\
\omega & a(\bar{\sigma})-\gamma
\end{array}\right)
$$


with $\vec{X}\left(t_{0}\right)=\vec{X}_{0}$ and $|\vec{X}|^{2}=X^{2}+Y^{2}$. All constants $\gamma, \tilde{\gamma}, \omega, \tau_{c}$ are to be specified in the simulations Section 4, while $a(\bar{\sigma})$ is defined in (2.15) below. Here $\bar{\sigma}$ denotes the area fraction as in (2.9) of the stochastic process $\sigma$. This is our main coupling variable of the ODE (2.2) with the stochastic noise model while the other one is the external field $h=h(\vec{X})$. The Jacobian of the linearized system (2.14) is,

$$
K=\frac{1}{\tau_{c}}\left[\begin{array}{cc}
a(\bar{\sigma})+\gamma & -\omega \\
\omega & a(\bar{\sigma})-\gamma
\end{array}\right]
$$

with eigenvalues

$$
\lambda=a(\bar{\sigma}) \pm i \sqrt{\omega^{2}-\gamma^{2}} .
$$

Oscillations occur for $\omega^{2}-\gamma^{2}>0$. In general however we observe convergence to a limit cycle for $a(\bar{\sigma}) \geq 0$ and decay to the stable node at $(0,0)$ otherwise.

In addition to the complex Ginzburg-Landau ODE we also consider scalar examples of ODE exhibiting bi-stability and saddle-node bifurcations. We summarize here the three types of examples which we will study in detail in the numerical investigations Section 4:

$$
\begin{aligned}
& \text { Scalar Bif.: } \quad f(X, \sigma)=a(\bar{\sigma}) X+\frac{\tilde{\gamma}}{\tau_{c}} X^{3}, \quad \text { where } a(\bar{\sigma})=\frac{1}{\tau_{c}}[b(1-\bar{\sigma})+z] \\
& \text { Saddle Bif.: } \quad f(X, \sigma)=a(\bar{\sigma})+\frac{\tilde{\gamma}}{\tau_{c}} X^{2}, \quad \text { where } a(\bar{\sigma})=\frac{b}{\tau_{c}}(z-\bar{\sigma}) \\
& \text { Hopf Bif.: } \quad f(\vec{X}, \sigma)=\frac{1}{\tau_{c}}\left[A \vec{X}-\tilde{\gamma}|\vec{X}|^{2} \vec{X}\right], \quad \text { where } a(\bar{\sigma})=b(\bar{\sigma}-z),
\end{aligned}
$$

for $\tilde{\gamma}, b, z$ constants varying per test case and matrix $A$ as in (2.14). Further details on all constants are also to be found in the numerical simulations Section 4 .

\section{Deterministic closures}

In this section we derive deterministic closures of the hybrid system (2.1), (2.2) in two distinct asymptotic regimes. Here we restrict our study to the spin flip case only. First, using stochastic averaging, we obtain an effective ODE for the variable $X=X(t)$ in the time-asymptotic limit $\tau_{I} \rightarrow 0$, i.e. when the relaxation time $\tau_{I}$ of the stochastic system (2.1) is much faster than the relaxation time $\tau_{c}$ of the ODE (2.2). The results and the accompanying simulations presented in this paper primarily refer to the case where phase transitions are absent in the spin flip system, although we briefly discuss the phase transition case also.

Second, we obtain a coupled, spatially distributed deterministic mesoscopic system for the evolution of $X$ and the average local coverage corresponding to the stochastic order parameter $\sigma$. Both derivations are carried out rigorously on a finite time interval $[0, T]$. Finally we briefly discuss the breakdown of the validity of the deterministic closures in infinite time intervals; more precisely, stochastic fluctuations can trigger large deviations from the averaged system given enough time, in which case the dynamic stability of the hybrid system may lead to an entirely different long-time behavior than the one predicted by the deterministic system. This issue, as well as the related issue of phase transitions in the spin flip system are fully explored in [8], however some relevant simulations are presented in Section 4.2, Table 4.2 and Figure 4.13 . 


\subsection{Stochastic averaging in time and the role of phase transitions.}

In the first deterministic closure example, we assume that the stochastic system evolves with a characteristic time which is much faster compared to that of the ODE. In this case we can apply a stochastic averaging principle, which is similar to the one presented in $[7]$ for systems of stochastic differential equations with fast and slow scales.

The main requirement here is the ergodicity property of the stochastic process $\left\{\sigma_{t}\right\}_{t \geq 0}$. Indeed, for any fixed, finite $N$, the process is ergodic with (2.5) as the unique invariant measure, [17]; in particular we have,

$$
\lim _{T \rightarrow \infty} \frac{1}{T} \int_{0}^{T} f\left(\vec{X}, \overline{\sigma_{t}}\right) d s=\bar{f}(\vec{X}) \quad \text { for } \quad \vec{X} \in R^{2}
$$

where

$$
\bar{f}(\vec{X})=E_{\mu_{\beta, N}} f(\vec{X}, \bar{\sigma}) \text { for } \quad \vec{X} \in R^{2} .
$$

Due to special structure of $f$ which depends on $\bar{\sigma}$ linearly (see (2.15)), we always have

$$
E_{\mu_{\beta, N}} f(\vec{X}, \bar{\sigma})=f\left(\vec{X}, u_{\beta, N}(h(\vec{X}))\right)
$$

where,

$$
u_{\beta, N}(h)=E_{\mu_{\beta, N}} \bar{\sigma}=\frac{1}{Z} \sum_{\{\sigma\}} \sum_{x \in \mathcal{L}} \sigma(x) e^{-\beta H(\sigma)} P_{N}(\sigma) .
$$

Furthermore (3.3) is known as average coverage or in classical statistical mechanics as average magnetization. It is clear from the formula that for finite $N, u_{\beta, N}(h)$ is an analytic function of $h$, we refer to [5] (Theorem V.4.3) for a detailed discussion of its properties (e.g. monotonically increasing, concave for $h>0$, symmetry in $h$, etc.). In other words there are no first-order phase transitions for finite $N$. In this case (3.2) and the averaged ODE (3.6) below are always well defined, regardless of temperature and strength of interactions. In fact we expect the following statement to hold:

- On an arbitrary bounded time interval $[0, T]$ with fixed $N$ and $\tau_{c}$ we have:

$$
\lim _{\tau_{I} \rightarrow 0} P\left(\sup _{0 \leq t \leq T}|\vec{X}(t)-\bar{x}(t)|>\delta\right)=0,
$$

for any $\delta>0$ where $\vec{X}=\vec{X}(t)$ is the solution of (2.2),

$$
\begin{cases}\frac{d}{d t} \vec{X} & =\frac{1}{\tau_{c}} f\left(\vec{X}, \sigma_{t}\right), \quad \text { for } \quad t \in[0, T] \\ \vec{X}_{0} & =x\end{cases}
$$

and $\bar{x}=\bar{x}(t)$ is the solution of the averaged system (depending on the lattice size $N)$

$$
\left\{\begin{array}{ll}
\frac{d}{d t} \bar{x}_{t} & =\frac{1}{\tau_{c}} \bar{f}\left(\bar{x}_{t}\right) \\
\bar{f}\left(\bar{x}_{t}\right) & =f\left(\bar{x}_{t}, u_{\beta, N}\left(h\left(\bar{x}_{t}\right)\right)\right) \\
\bar{x}_{0} & =x
\end{array} \quad \text { for } t \in[0, T] .\right.
$$


REMARK 3.1. The main difference and difficulty in the rigorous proof of this statement over the analogous one in [7] is that in contrast to the SDEs discussed there, here the original process (2.1), (2.2) is a jump process, so continuity arguments such as (9.5), (9.6) in [7] do not apply directly. To that end it may be possible to adapt the methods of [7] along with an asymptotic expansion of the generator in (2.1).

REMARK 3.2. As pointed out earlier, for a finite $N$, the spin flip system has, strictly speaking, no phase transitions which can arise depending on the competition between fluctuations and interactions, in the $N \rightarrow \infty$ limit. Therefore (3.4) always applies since in that context $N$ is kept fixed, while $\tau_{I} \rightarrow 0\left(\tau=\tau_{c} / \tau_{I} \rightarrow \infty\right)$. However, even for finite $N$, phase transitions are manifested in hysteresis $M C$ simulations (see for instance Figures 1 and 2 in [9]). We expect a similar phenomenon to arise here, since the coupling of (2.2) to the spin flip system is through the external field $h=h(\vec{X})$.

In this paper we restrict our study to regimes that do not have phase transitions, in which case the formulation of (3.6) is unambiguous. We return to the study of phase transitions in a subsequent publication [8] where metastability and hysteresis phenomena dominate the coupled system, giving rise to multiple steady states, excitable states and oscillations.

We conclude this section by noting that there are two practical ways to obtain and implement the averaged system (3.6) by first obtaining $u_{\beta, N}(h)$.

In general we can calculate $u_{\beta, N}(h)$ numerically. That is for a given $h$ we calculate by Monte Carlo $E_{\mu_{\beta, N}} \bar{\sigma} \rightarrow u_{\beta, N}(h)$ as $t \rightarrow \infty$. As discussed earlier for a fixed number $N$ of lattice sites and periodic boundary conditions, the microscopic system does not exhibit phase transitions, hence the aforementioned limits are well defined. In this case there are no restrictions on the range of interactions. In fact in the nearest neighbor case $\lim _{N \rightarrow \infty} u_{\beta, N}(h)=u_{\beta}(h)$ can be calculated explicitly (pg. 49, [15])

$$
u_{\beta}(h)=\frac{1}{2}-\frac{\sinh (\beta h)}{2 \sqrt{\sinh ^{2}(\beta h)+\exp \left(-4 \beta J_{0}\right)}}
$$

which is analogous to Figure 3.1 below.

In the long range interaction limit $N=2 L+1, L, N \rightarrow \infty$, we obtain the usual mean-field formulae where $u_{\beta}(h)=\lim _{N \rightarrow \infty} u_{\beta, N}(h)$ is the unique minimizer $(h \neq 0)$ of the free energy functional, [22]

$$
I[c]=\beta h c+r(c)-\beta \frac{J_{0}}{2} c^{2}
$$

where $r(c)=c \log c+(1-c) \log (1-c)$ and $c$ corresponds to average magnetization. We therefore solve the following nonlinear equation for $u_{\beta}$ :

$$
h=J_{0} u_{\beta}-\frac{1}{\beta} \log \frac{u_{\beta}}{1-u_{\beta}} .
$$

We give such a calculation in Figure 3.1 for different values of $\beta$. There we plot the minimizer $u_{\beta}$ of (3.7) as a function of the external field $h$. Beyond the phase transition point (which is nontrivial even in one-dimensional mean-field models), i.e. for $\beta J_{0}>4$, $u_{\beta}$ is a multivalued function of $h$, see Fig 3.1. Numerically we have observed that for $L>20$ and $N=1000$ lattice nodes $u_{\beta, N}(h)$ is satisfactorily approximated by $u_{\beta}(h)$ from (3.8).

In Figure 3.2 we present numerical Monte Carlo simulations of $u_{\beta, N}(h)$ for different radius potential lengths and contrast them with the exact analytic solution 
The Free Energy Minimizer $u_{\beta}(h)$

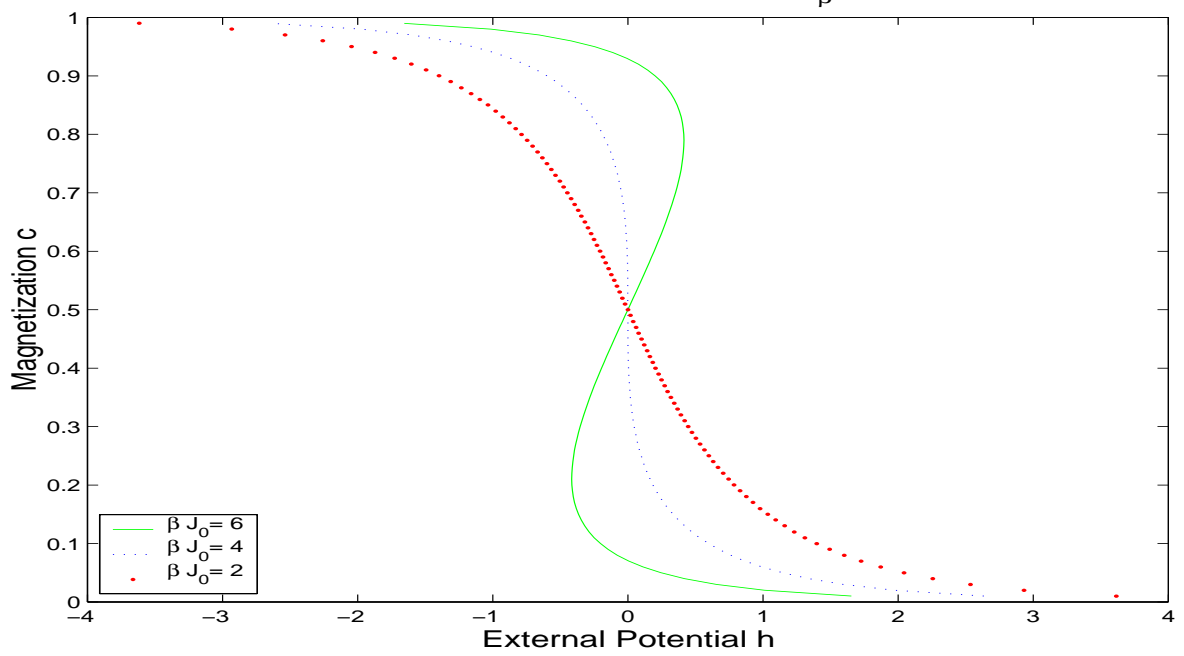

FIG. 3.1. The minimizer $u_{\beta}(h)$ of (3.7) for the cases of $\beta J_{0}=6,4$ and 2. A phase transition occurs for $\beta J_{0}>4$.

$u_{\beta}(h)$ from (3.8). We plot the comparisons for the case of $\beta J_{0}=2$. Note that even for very few spline nodes we have good agreement with the analytically calculated minimizer of (3.8) regardless of radius length. Hence, in the remainder of the paper we implement $u_{\beta, N}(h)$ by approximating it by $u_{\beta}(h)$ given by (3.8) but note that in a few examples we observe some systematic errors due to this approximation.

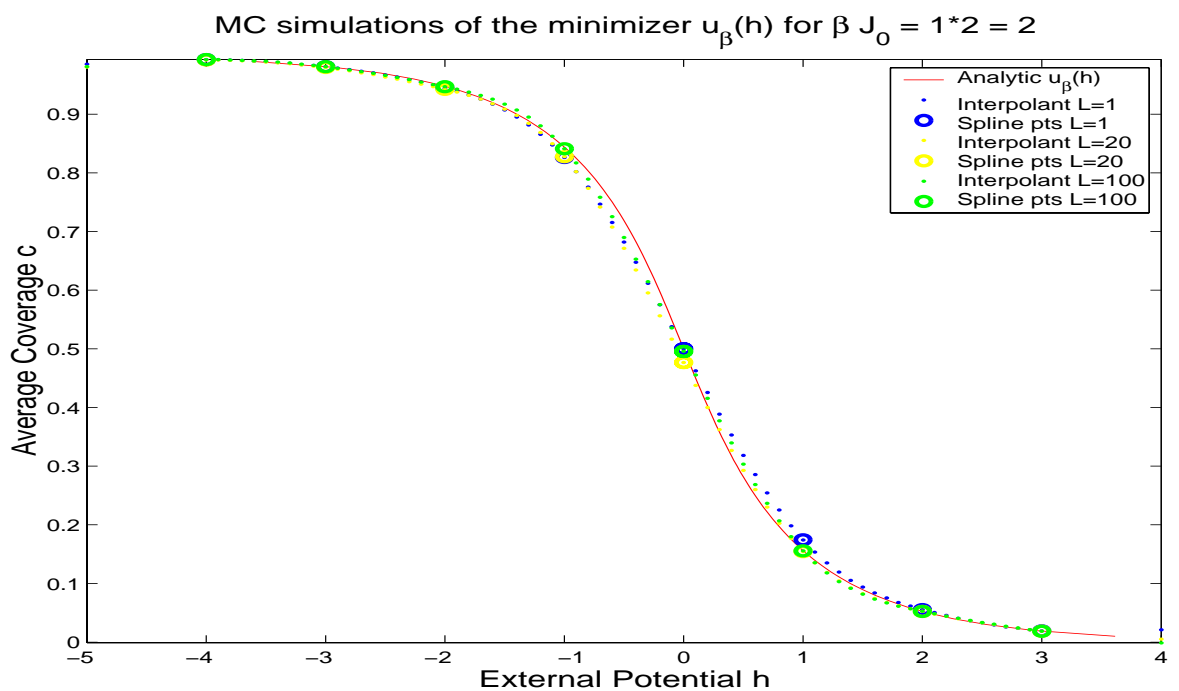

FIG. 3.2. Analytic solution of (3.8) compared to numerical MC implementations at different potential radius lengths. We plot both the spline points as well as the splines through those points. 
3.2. Deterministic closures in a general setting. We now attempt to carry out the derivation of mean-field equations for our coupled system (2.1), (2.2) without the time-scales separation assumed in the previous section, by writing an evolution equation for the mean values of the random processes. As in similar derivations for other microscopic systems such as the Boltzmann equation, it is expected that this will not be possible in general, due to the fact that the evolution of any single moment of the random process will likely depend on higher moments, creating an infinite hierarchy of equations. To demonstrate this fact we will next derive one such exact but not closed equation for the simplest observable, namely the average coverage $E \sigma(x)$.

We consider the spin flip Arrhenius type dynamics which we presented in Section 2.1 , and using the definition of the generator, we obtain the following time evolution law for the average coverage $E \sigma(x)$ :

$$
\frac{d}{d t} E \sigma(x)=E(1-2 \sigma(x)) c(x, \sigma),
$$

where $c(x, \sigma)$ is given in (2.10). Therefore,

$$
\begin{gathered}
\frac{d}{d t} E \sigma(x)=E(1-2 \sigma(x))\left(c_{a}(1-\sigma(x))+c_{d} \sigma(x) e^{-\beta U(x)}\right) \\
=E\left(c_{a}-c_{a} \sigma(x)+c_{d} \sigma(x) e^{-\beta U(x)}-2 \sigma(x) c_{a}\right. \\
\left.\quad+2 \sigma^{2}(x) c_{a}-2 c_{d} \sigma^{2}(x) e^{-\beta U(x)}\right)
\end{gathered}
$$

with $U(x ; \vec{X}, \sigma)$,

$$
U(x ; \vec{X}, \sigma)=\sum_{z \neq x} J(x, z) \sigma(z)-h(\vec{X}) .
$$

Note here that,

$$
\sigma^{2}(x)=\sigma(x) \text { and therefore also, } E \sigma^{2}(x)=E \sigma(x) ;
$$

recalling that $c_{a}=c_{d}=\frac{1}{\tau_{I}}$ we obtain

$$
\frac{d}{d t} E \sigma(x)=\frac{1}{\tau_{I}} E\left[(1-\sigma(x))-\sigma(x) e^{-\beta U(x ; \vec{X}, \sigma)}\right]
$$

Note that (3.10) is exact but not closed in terms of $E \sigma(x)$. By carrying out a spatial average over (3.10) we can also obtain an exact but still not closed formula for $E \bar{\sigma}$. Although such an equation cannot provide a predictive tool by itself, it can certainly be used, since it is exact, as a numerical benchmark for the deterministic and stochastic closures presented next.

3.3. Deterministic mesoscopic closures. In this subsection we obtain in the asymptotic limit of long range interactions a spatially distributed deterministic closure of (2.1), (2.2), without the time-scales separation assumed in the previous section.

We discuss first formally, and in the spirit of the intuitive derivations in [23] for uncoupled spin flip models, exact closures for $E \sigma_{t}(x)$ in the case of weak long range interactions, i.e. when $N=2 L+1, N, L \rightarrow \infty$. As in [23] we assume "propagation of chaos" for the microscopic system, in which case the fluctuations of the spins 
$\{\sigma(x), x \in \mathcal{L}\}$ about their mean values are independent and the law of large numbers formally applies. Thus the fluctuations of $\beta \sum_{y \neq x} J(x, y) \sigma(y)$ about its mean will be small such that in the long range interaction limit we will have,

$$
E e^{-\beta \sum_{y \neq x} J(x, y) \sigma(y)}=e^{-\beta \sum_{y \neq x} J(x, y) E \sigma(y)}+o_{N}(1) .
$$

Therefore we formally obtain from (3.10),

$$
\left.\frac{d}{d t} E \sigma(x)=\frac{1}{\tau_{I}}\{1-E \sigma(x))-E\left(\sigma(x) e^{\beta h(\vec{X})}\right) e^{-\beta \sum_{y \neq x} J(x, y) E \sigma(y)}\right\}+o_{N}(1) .
$$

We denote the local average coverage,

$$
\begin{aligned}
& u(x, t)=E \sigma_{t}(x), \quad v(x, t)=E U(x)=\beta \sum_{y \neq x} J(x, y) u(y, t) \\
& \quad \text { and } \quad \vec{Y}=E \vec{X}
\end{aligned}
$$

and we obtain the system of equations,

$$
\begin{cases}\frac{d}{d t} \vec{Y} & =\frac{1}{\tau_{c}} f(\vec{Y}, \bar{u}) \\ \frac{d}{d t} u(x, t) & \left.=\frac{1}{\tau_{I}}(1-u(x, t))-e^{-v(x, t)} E \sigma(x) e^{\beta h(\vec{X})}\right)+o_{N}(1) \quad \text { for each } x \in \mathcal{Z},\end{cases}
$$

where $\bar{u}(t)=\frac{1}{N} \sum_{x} u(x, t)$. At this point it is not obvious when this system can be closed, even as $N \rightarrow \infty$, since the dependence of the external potential $h$ on the random process $\vec{X}$ makes it unclear how to pass the expected value inside in the product, $E \sigma(x) e^{\beta h(\vec{X})}$ without the use of further simplifying assumptions.

However on this issue the rigorous derivation of mean-field equations is more systematic and illuminating. As in the uncoupled case, instead of writing an equation directly for $E \sigma_{t}(x)$, we consider an averaged - but still stochastic - quantity, namely the empirical measure,

$$
\mu^{N}(d y, t)=\frac{1}{N} \sum_{x \in \mathcal{L}} \sigma_{t}(x) \delta_{x}(d y), \quad \text { for } \quad y \in R
$$

In this case (2.2) becomes exactly,

$$
\frac{d \vec{X}}{d t}=f\left(\vec{X}, \bar{\mu}^{N}\right) \quad \text { with } \quad \bar{\mu}^{N}(t)=\int_{R} \mu^{N}(d z, \sigma)=\bar{\sigma}_{t},
$$

while (as we will show in the proof below) for the stochastic model we have,

$$
\begin{aligned}
\frac{d}{d t}<\mu^{N}(\cdot, t), \phi> & =\frac{c_{a}}{N} \sum_{x \in \mathcal{L}} \phi(x)-c_{a}<\mu^{N}(\cdot, t), \phi> \\
& -c_{d}<\mu^{N}(\cdot, t), \phi \exp \left[-\beta\left(J * \mu^{N}(\cdot, t)-h(\vec{X})\right)\right]+o_{N}(1) .
\end{aligned}
$$

Passing to the weak-* limit in $\mu^{N}(d y, t)$ we have:

THEOREM 3.3. If the potential $J(x, y)=\frac{1}{2 L+1} J\left(\frac{|x-y|}{2 L+1}\right)$ is smooth long-ranged, i.e. $N=2 L+1$, then for any $T$ finite we have that

$$
\lim _{N \rightarrow \infty} X(t)=Y(t), \quad \text { uniformly in } \quad[0, T],
$$


and

$$
\lim _{N \rightarrow \infty} \mu^{N}(d y, t)=u(y, t) d y, \quad \text { uniformly in } \quad[0, T],
$$

where the latter limit is in the sense of probability measures, and $(Y, u)$ solve (compare to (3.11)),

$$
\left\{\begin{aligned}
\frac{d}{d t} \vec{Y} & =\frac{1}{\tau_{c}} f(\vec{Y}, \bar{u}) \\
\frac{d}{d t} u & =\frac{1}{\tau_{I}}\left\{1-u-u e^{-\beta J * u} e^{\beta h(\vec{Y})}\right\} \quad \text { for } t \in[0, T]
\end{aligned}\right.
$$

where $\bar{u}(t)=\int_{0}^{1} u(x, t) d x$.

Proof: We consider the empirical measure $\mu^{N}(d y ; t)=\frac{1}{N} \sum_{x \in \mathcal{L}} \sigma_{t}(x) \delta_{x}(d y)$ as our observable quantity. Then we define

$$
f(\sigma)=<\mu^{N}, \phi>=\frac{1}{N} \sum_{x \in \mathcal{L}} \sigma_{t}(x) \phi(x)
$$

for any test function $\phi$, and consider the martingale

$$
M_{t}=f\left(\sigma_{t}\right)-f\left(\sigma_{0}\right)-\int_{0}^{t} L f\left(\sigma_{s}\right) d s
$$

with quadratic variation

$$
<M_{t}>=\int_{0}^{t} L f^{2}\left(\sigma_{s}\right)-2 f\left(\sigma_{s}\right) L f\left(\sigma_{s}\right) d s=\frac{2}{N^{2}} \int_{0}^{t} \sum_{x \in \mathcal{L}}[c(x, \sigma)] \phi^{2}(x),
$$

hence $E<M_{t}>=O(1 / N)$. By Doob's maximal inequality we have that for any time horizon $t_{1}$,

$$
P\left(\sup _{t \in\left[0, t_{1}\right]}\left|M_{t}\right|>\delta\right) \leq \frac{1}{\delta^{2}} O(1 / N) .
$$

Thus on a set of probability approximately one we have,

$$
<\mu^{N}(\cdot, t), \phi>=<\mu^{N}(\cdot, 0), \phi>+\int_{0}^{t} L<\mu^{N}(\cdot, s), \phi>d s+O(\delta),
$$

where a short calculation shows that

$$
\begin{aligned}
L<\mu^{N}(\cdot, s), \phi> & =\frac{d_{0}}{m} \sum_{x \in \mathcal{L}} \phi(x)-d_{0}<\mu^{N}(\cdot, s), \phi> \\
& -d_{0}<\mu^{N}(\cdot, s), \phi \exp \left[-\beta\left(\bar{J} * \mu^{N}-h(\vec{X})\right)\right]>,
\end{aligned}
$$

and $\vec{X}=\vec{X}(t)$ solves (3.12).

Here we remark that the assumption of long-range interactions allowed us to rewrite the right-hand side of (3.14) as a function of $\mu^{N}(d y, s)$ and thus obtain an approximate closed equation for the measure $\mu^{N}(d y, s)$. The relative compactness of the probability distributions of the random measures $\mu^{N}(d x, t)$ in the space $D\left([0, T], \mathcal{M}_{+}\right)$ 
(the set of right continuous functions with left limits taking values in the space of positive finite measures $\mathcal{M}_{+}$) follows from the estimate on the quadratic variation $\left\langle M_{t}\right\rangle$. Then passing to the $N \rightarrow \infty$ limit in (3.14) and (3.12) we obtain

$$
\begin{aligned}
<\mu(\cdot, t), \phi>-<\mu(\cdot, 0), \phi> & =\int_{0}^{t} d_{0}<\lambda-\mu(\cdot, s), \phi> \\
& -d_{0}<\mu(\cdot, s), \phi \exp [-\beta(J * \mu-h(Y(t)))] d s,
\end{aligned}
$$

and

$$
\frac{d \vec{Y}}{d t}=f(\vec{Y}, \bar{\mu}) \quad \text { with } \quad \bar{\mu}(t)=\int_{R} \mu(d y, t),
$$

where $\lambda$ is the Lebesgue measure in $T$. It is not hard to see that the measure valued solutions are absolutely continuous with respect to the Lebesgue measure, i.e. $\mu(d x, t)=u(x, t) d x$ (we refer to [14] for similar compactness and regularity arguments) thus the theorem follows in the strong sense.

REMARK 3.4. To obtain stochastic corrections to the mean-field equation (3.13), we can formally apply the expansion,

$$
\begin{aligned}
\vec{X}(t) & =\vec{Y}(t)+\frac{1}{\sqrt{N}} \zeta \\
\mu^{N}(d y, t) & =u(\xi, t) d y+\frac{1}{\sqrt{N}} \xi
\end{aligned}
$$

where $u=E \sigma_{t}$ and $(\vec{Y}, u)$ are deterministic averages and $(\zeta, \xi)$ central limit theorem type corrections. We obtain a system where $(Y, u)$ satisfies the mean field system (3.11), while the stochastic corrections $(\zeta, \xi)$ satisfy respectively a linearized ODE derived from (2.2) and a linear (linearized according to (3.15)) Stochastic Partial Differential Equation (SPDE) for $\xi$ (see for instance [4] and [21] for the uncoupled case). These derivations are typically valid only for finite time intervals $[0, T]$. We also refer to the corresponding results for SDE asymptotics in Section 4 of [7]. Therefore (3.13) and the corresponding SPDE for $\xi$ may hold globally in time only under special conditions on their asymptotics at long times e.g a stable limit point or a stable limit cycle for (3.13). Otherwise the expansions in (3.15) (and their consequences) cannot be reliable. In this case the proper asymptotics are given by large deviations and WKB expansions (see the first case of Table 4.2, the third case of Table 4.1 and the cases for $\tau_{c}=.1$ in Tables 4.3-4.3).

\section{Numerical simulations}

We now present a number of numerical comparisons based on Monte Carlo simulations of the full coupled system $(2.1,2.2)$ against the numerical solution of the reduced ODE (3.6) as obtained by averaging principle theory (for $\tau=\tau_{c} / \tau_{I} \rightarrow \infty$ ). However we also include examples which are not in the $\tau>>1$ regime so as to further understand the extent of validity of the theoretical predictions.

Here we only carry out and present simulations and comparisons for the averaging principle system (3.6). We will not present any comparisons in this work between (2.1, 2.2 ) and the mean field theory closures (3.13) but instead defer this presentation to a future work [8] (there we also focus on the phase transition regime where spatially distributed models are of greater significance).

The hybrid system is comprised of a microscopic dynamics stochastic model (2.1) in $\sigma$, coupled to an ODE of the form (2.15). Note that the ODE is coupled to the 
stochastic by parameterizing one of its coefficients through the noise parameter,

$$
\bar{\sigma}=\frac{1}{N} \sum_{x \in \mathcal{L}} \sigma .
$$

On the other hand, the stochastic system is coupled to the ODE through the application of an external potential $h \equiv h(\vec{X})$. We choose a scalar dependence for our external potential of either linear or quadratic type as follows,

$$
h(\vec{X})=c X+h_{0}, \quad \text { or } \quad h(\vec{X})=c X^{2}+h_{0}
$$

for appropriate constants $c$ and $h_{0}$ whose values are specified for each case examined below. We summarize the coupling in the following diagram,

\begin{tabular}{|c||c|c|}
\hline & ODE & Stochastic Model \\
\hline \hline Requires & $\vec{X}, \bar{\sigma}(x)$ & $\sigma(x), h(\vec{X})$ \\
\hline Provides & $\vec{X}$ & $\sigma(x)$ \\
\hline
\end{tabular}

Each equation $(2.3,2.15)$ comprising system $(2.1,2.2)$ has its own time scale,

$$
\begin{aligned}
& \tau_{c} \Rightarrow \text { characteristic time for ODE } \\
& \tau_{I} \Rightarrow \text { characteristic time for stochastic spin dynamics. }
\end{aligned}
$$

We define

$$
\tau=\tau_{c} / \tau_{I}
$$

and explore the following three cases:

- $\tau>>1 \Longrightarrow$ ODE equilibrates slower than the stochastic spin dynamics.

- $\tau \approx 1 \Longrightarrow$ ODE equilibrates at similar times as the spin dynamics.

- $\tau<<1 \Longrightarrow$ ODE equilibrates faster than the spin dynamics.

where without loss of generality we fix $\tau_{I} \equiv 1$ and vary $\tau_{c}$ accordingly for the cases specified above.

We monitor how the phase portrait of our dynamical system $(2.1,2.2)$ transforms when the critical parameter coupled to $\bar{\sigma}$ passes through its bifurcating value. Furthermore we compare how this behavior agrees or not with our averaging principle theory. We perform these numerical comparisons under the three cases of $\tau_{c}$ outlined above and a variety of values for the fixed parameters of the external potential and the ODEs corresponding to a wealth of different phase space profiles.

The full coupled system is numerically solved by performing both, a Monte Carlo simulation for (2.1) and an adaptive ODE solver for (2.2) in parallel. Each iteration of the Monte Carlo simulation produces a variable time step $\Delta t$ and is immediately followed by applying the ODE solver. The solver iterates until the given time step $\Delta t$ has been exhausted. This procedure repeats until the stopping criteria (see below) have been met. More specifically we obtain a solution of (2.1) by implementing the following process-type kinetic Monte Carlo (KMC) algorithm [3] for spin flip Arrhenius dynamics:

Spin Flip Kinetic Monte Carlo Pseudo-code (Global Update Scheme)

1. Calculate all transition rates $c_{a}(l, \sigma)$ (absorption), $c_{d}(l, \sigma)$ (desorption), from (2.10) for all nodes $l$ in the lattice $\mathcal{L}$.

2. Calculate the total $R_{a}=\sum_{l \in \mathcal{L}} c_{a}(l, \sigma), R_{d}=\sum_{l \in \mathcal{L}} c_{d}(l, \sigma)$ absorption, desorption rates respectively. Obtain the total rate $R_{T}=R_{a}+R_{d}$ based on above. 
3. Obtain two random numbers, $\rho_{1}$ and $\rho_{2}$.

4. Use the first random number to choose between absorption or desorption based on the measure created by the rates $R_{a}, R_{d}$ and $R_{T}$. Let us assume that the choice is to absorb(desorb). Then we denote $c \equiv c_{a}(l, \sigma)\left(c_{d}(l, \sigma)\right)$ and $R=R_{a}\left(R_{d}\right)$.

5. Find the node at lattice position $k \in \mathcal{L}$ such that,

$$
\sum_{j=0}^{k} c(j, \sigma) \geq \rho_{2} R>\sum_{j=0}^{k-1} c(j, \sigma)
$$

6. Update the time, $t=t+\Delta t$ where $\Delta t=1 / R_{T}$.

7. Repeat from step 1 until equilibration or dynamics of interest have been captured.

As expected a kinetic Monte Carlo algorithm produces no "null" steps and therefore every iteration is a success. (A version of the pseudo-code just described has also been created which implements a local update scheme at every iteration thus improving speed at the reciprocal expense of allocating more computer memory).

We follow-up each iteration of our stochastic solver by implementing a solution of the ODE (2.15) for the time step $\Delta t$. We employ a 4th order adaptive RungeKutta-Fehlberg (RKF). The scheme applies a Runge-Kutta method of order 5 to estimate the error in a Runge-Kutta method of order 4. Further the scheme enforces a computational advantage of performing only six evaluations per time step instead of eleven. At each iteration, the time step is adjusted up or down to control the error. We use the same RKF ODE solver to also obtain a solution of the resulting reduced averaging principle ODE (3.6).

We use a finite size interaction potential range $L<\infty$. In all simulations in this work we set $N=1000$ lattice nodes and a potential radius of $L=20$. Note that in (3.6) $u_{\beta, N}(h)$ is obtained from the mean field formula (3.8) since as we demonstrated (see Figure 3.2) there is good agreement in the absence of phase transitions.

Below we display a number of examples from our Monte Carlo simulations versus the reduced average principle equation for the three equations presented in (2.15) which display the following stability behaviors:
(1) Scalar Bifurcation
(2) Saddle Bifurcation
(3) Hopf Bifurcation For each case we present in full detail both, the complete ODE and its corresponding averaging principle system based on the theory developed in Section 3. After each such case we display selected simulation results and other statistical comparisons in tables and figures. In general the tables amass succinct statistical comparisons while the selected figures display interesting behavior of the systems in terms of stability tendencies, potentials, probability density functions etc. The numerical data presented in this report which validate our claims are only but a fraction of the several hundreds of numerical simulations undertaken.

4.1. Scalar bifurcation. We start by studying one of the most fundamental dynamical systems, a scalar (pitchfork) bifurcation. We therefore set (2.2) for $\vec{X}=X$ as follows,

$$
\frac{d X}{d t}=a(\bar{\sigma}) X+\gamma X^{3}
$$

where we let

$$
a(\bar{\sigma})=\frac{1}{\tau_{c}}[z+(1-\bar{\sigma}) b]
$$


and $\gamma=\tilde{\gamma} / \tau_{c}$ with $b=4, z=-1$ and $\tilde{\gamma}= \pm .025$. The external potential we apply in this example is linear and takes the form,

$$
h=h(X)=c X+h_{0}
$$

with $c=-1, h_{0}=1$. Note that (4.2) has three nodes: $0, \pm \sqrt{-a(\sigma) / \gamma}$ and two main bifurcation states: super-critical, in the case of a stable node turning into one unstable and two stable nodes and sub-critical in the opposite situation.

The corresponding averaged system for (2.1) based on the form of (4.2) reduces to,

$$
\begin{cases}\frac{d}{d t} \bar{x} & =\frac{1}{\tau_{c}}\left[b\left(1-u_{\beta, N}(h(\bar{x}))+z\right] \bar{x}+\frac{\tilde{\gamma}}{\tau_{c}} \bar{x}^{3}\right. \\ \bar{x}_{0} & =x\end{cases}
$$

Recall that following the discussion in Section 3.1, $u_{\beta, N}$ is approximated by the minimizer of (3.7).

We present two numerical comparisons between the Monte Carlo implementations of (4.2) and its averaging principle analogue (4.3). We start with a super-critical bifurcation set-up by setting $\tilde{\gamma}=-.025$ and we display the results in Table 4.1 for the regime of $\tau_{c}=5$ and 1 where averaging principle theory is expected to be valid. As expected we have agreement between both the Monte Carlo simulations based on (4.2, 2.1) and averaging principle solutions corresponding to (4.3). We display the Monte Carlo solutions for $X$ and $\bar{\sigma}$ in Figure 4.1 for the case of $\beta J_{0}=.01$ where typically the noise levels are highest. Note however that the noise is not strong enough for the solution to perform the "jump" from the current node at $X=1.97$ to the node at $-\infty$ as can be seen in Figure 4.2. In general the effect of noise is most noticeable when the wells are shallower. This point will become clear in the next few examples.

In the second example we display results of comparisons performed for all cases of $\tau_{c}=.1,1$ and 5 and change $\tilde{\gamma}=.025$ (sub-critical bifurcation set-up). Note that at least for the case of $\tau_{c}=.1$ we do not expect, at least in theory, agreement between the Monte Carlo simulations and our averaging principle reduced equation. The results for $\tau_{c}=.1,1$ and 5 are displayed in Tables, 4.1, 4.1 and 4.1 respectively. Remarkably, we seem to have agreement for all cases examined; even for the case of $\tau_{c}=.1$. A typical Monte Carlo solution is displayed for $\beta J_{0}=.01$ in Figure 4.3 where we observe the solution accumulating around the node at $X \approx 2.28$. The respective potential well is shown in Figure 4.4. Notice that comparatively the potential well in this figure is even higher than the one in Figure 4.2 of our first example. This may account for the fact of not observing the expected disagreement between the Monte Carlo simulations and the averaging principle solutions. However this will not always be the case as will be seen in the examples to follow.

Note that the highest relative error is observed for the case $\beta J_{0}=2$ in all Tables 4.1-4.1. This is expected however since for $\bar{\sigma}=.75$ we have $a(\bar{\sigma})=0$ in (4.2) thus triggering a change in the ODE stability profile and creating a bifurcation from one node to three nodes and visa versa. Since our system hovers around this critical value our predictions are understandably less accurate in that region. 
TABLE 4.1. Example 1. Monte Carlo simulations versus averaging principle predictions for scalar bifurcation. MC statistics from a data set comprised of 100,000 samples. Results shown for $\tau=1,5$. This is the case where we expect averaging principle theory to be valid. As can be seen from the results and the analysis we have complete agreement.

\begin{tabular}{|c|c||cccc|}
\hline \multirow{2}{*}{$\beta J_{0}$} & Char. & \multicolumn{4}{c|}{ Monte Carlo Simulations Summary Statistics } \\
& time & $\bar{X}$ & $\operatorname{StDev}(\mathrm{X})$ & $\overline{\bar{\sigma}}$ & $\operatorname{StDev}(\bar{\sigma})$ \\
\hline \hline .01 & $\tau=1$ & 1.9669 & .008 & .7258 & .014 \\
\hline .01 & $\tau=5$ & 1.9666 & .003 & .7258 & .014 \\
\hline 2 & $\tau=5$ & 0.6336 & .002 & .7476 & .006 \\
\hline-2 & $\tau=5$ & 3.1395 & .003 & .6884 & .012 \\
\hline
\end{tabular}

\begin{tabular}{|c|c||cc|}
\hline \multirow{2}{*}{$\beta J_{0}$} & $\begin{array}{c}\text { Char. } \\
\text { time }\end{array}$ & \multicolumn{2}{|c|}{$\begin{array}{c}\text { Averaging Principle Statistics } \\
\bar{X}\end{array}$} \\
\hline \hline .01 & $\tau=1$ & 1.9727 & .7256 \\
\hline .01 & $\tau=5$ & 1.9727 & .7256 \\
\hline 2 & $\tau=5$ & .7034 & .7469 \\
\hline-2 & $\tau=5$ & 3.0837 & .6905 \\
\hline
\end{tabular}

Analysis for $\tau_{c}=5$ :

\begin{tabular}{lcccc} 
Case & Characterization & Aver. Princ. & MC Simul. & Rel. Error \\
\hline$\beta J_{0}=.01$ & stable node at & $\bar{X}_{A P}=1.9727$ & $\bar{X}_{M C}=1.9669$ & 0.003 \\
$\beta J_{0}=2$. & stable node at & $\bar{X}_{A P}=.7034$ & $\bar{X}_{M C}=.6336$ & .110 \\
$\beta J_{0}=-2$. & stable node at & $\bar{X}_{A P}=3.0837$ & $\bar{X}_{M C}=3.1395$ & .018
\end{tabular}

TABLE 4.2. Example 2. Monte Carlo simulation versus averaging principle predictions for scalar bifurcation. Characteristic time here is set to $\tau=.1$. This is the regime where we would not expect agreement in the results. Nevertheless we do observe agreement!

\begin{tabular}{|c|c|c|c|c|}
\hline \multirow{2}{*}{$\beta J_{0}$} & \multicolumn{4}{|c|}{ Monte Carlo Simulations Summary Statistics } \\
\hline & $\bar{X}$ & $\operatorname{StDev}(X)$ & $\mathrm{St}$ & $\operatorname{ev}(\bar{\sigma})$ \\
\hline .01 & 2.2903 & .031 & .7828 & .013 \\
\hline 2 & 0.6506 & .019 & .7526 & .017 \\
\hline-2 & $+\infty$ & - & 1. & \\
\hline & $\beta J_{0}$ & $\begin{array}{l}\text { Averaging } \mathrm{Pr} \\
\bar{X}\end{array}$ & $\begin{array}{c}\text { siple Statistics } \\
\bar{u}_{\beta}\end{array}$ & \\
\hline & .01 & 2.2802 & .7825 & \\
\hline & 2 & .7255 & .7533 & \\
\hline & -2 & $+\infty$ & 1.0000 & \\
\hline
\end{tabular}

Analysis for $\tau_{c}=.1$ :

\begin{tabular}{lcclc} 
Case & Characterization & Aver. Princ. & MC Simul. & Rel. Error \\
\hline$\beta J_{0}=.01$ & stable node at & $\bar{X}_{A P}=2.2802$ & $\bar{X}_{M C}=2.2903$ & .004 \\
$\beta J_{0}=2$. & stable node at & $\bar{X}_{A P}=.7255$ & $\bar{X}_{M C}=.6506$ & .103 \\
$\beta J_{0}=-2$. & stable node at & $\bar{X}_{A P}=+\infty$ & $\bar{X}_{M C}=+\infty$ & -
\end{tabular}


TABlE 4.3. Example2. Monte Carlo simulations versus averaging principle predictions for scalar bifurcation. Characteristic time here is set to $\tau=1$. Once again based on the results we observe complete agreement between the solutions.

\begin{tabular}{|c||cccc|}
\hline \multirow{2}{*}{$\beta J_{0}$} & \multicolumn{4}{|c|}{ Monte Carlo Simulations Summary Statistics } \\
& $\bar{X}$ & $\operatorname{StDev}(X)$ & $\overline{\bar{\sigma}}$ & $\operatorname{StDev}(\bar{\sigma})$ \\
\hline \hline .01 & 2.2710 & .010 & .7822 & .013 \\
\hline 2 & 0.6502 & .006 & .7526 & .010 \\
\hline-2 & $+\infty$ & - & 1. & - \\
\hline
\end{tabular}

\begin{tabular}{|c||cc|}
\hline$\beta J_{0}$ & \multicolumn{2}{|c|}{ Averaging Principle Statistics } \\
& $\bar{X}$ & $\bar{u}_{\beta}$ \\
\hline \hline .01 & 2.2803 & .7825 \\
\hline 2 & .7255 & .7533 \\
\hline-2 & $+\infty$ & 1.0000 \\
\hline
\end{tabular}

Analysis for $\tau_{c}=1$ :

\begin{tabular}{lcccc} 
Case & Characterization & Aver. Princ. & MC Simul. & Rel. Error \\
\hline$\beta J_{0}=.01$ & stable node at & $\bar{X}_{A P}=2.2803$ & $\bar{X}_{M C}=2.2710$ & 0.004 \\
$\beta J_{0}=2$. & stable node at & $\bar{X}_{A P}=.7255$ & $\bar{X}_{M C}=.6502$ & .104 \\
$\beta J_{0}=-2$. & stable node at & $\bar{X}_{A P}=+\infty$ & $\bar{X}_{M C}=+\infty$ & -
\end{tabular}

TABLE 4.4. Example 2. Monte Carlo simulations versus averaging principle predictions for scalar bifurcation example 2. Characteristic time here is set to $\tau=5$. In this case we would expect agreement of results.

\begin{tabular}{|c||cccc|}
\hline \multirow{2}{*}{$\beta J_{0}$} & \multicolumn{4}{c|}{ Monte Carlo Simulations Summary Statistics } \\
& $\bar{X}$ & $\operatorname{StDev}(X)$ & $\overline{\bar{\sigma}}$ & $\operatorname{StDev}(\bar{\sigma})$ \\
\hline \hline .01 & 2.2711 & .005 & .7821 & .013 \\
\hline 2 & 0.6511 & .003 & .7529 & .017 \\
\hline-2 & $+\infty$ & - & 1. & - \\
\hline
\end{tabular}

\begin{tabular}{|c||cc|}
\hline \multirow{2}{*}{$\beta J_{0}$} & \multicolumn{2}{|c|}{ Averaging Principle Statistics } \\
& $\bar{X}$ & $\bar{u}_{\beta}$ \\
\hline \hline .01 & 2.2802 & .7825 \\
\hline 2 & .7255 & .7533 \\
\hline-2 & $+\infty$ & 1.0000 \\
\hline
\end{tabular}

Analysis for $\tau_{c}=5$ :

\begin{tabular}{lcccc} 
Case & Characterization & Aver. Princ. & MC Simul. & Rel. Error \\
\hline$\beta J_{0}=.01$ & stable node at & $\bar{X}_{A P}=2.2802$ & $\bar{X}_{M C}=2.2711$ & 0.0040 \\
$\beta J_{0}=2$. & stable node at & $\bar{X}_{A P}=.7255$ & $\bar{X}_{M C}=.6511$ & .1055 \\
$\beta J_{0}=-2$. & stable node at & $\bar{X}_{A P}=+\infty$ & $\bar{X}_{M C}=+\infty$ & -
\end{tabular}



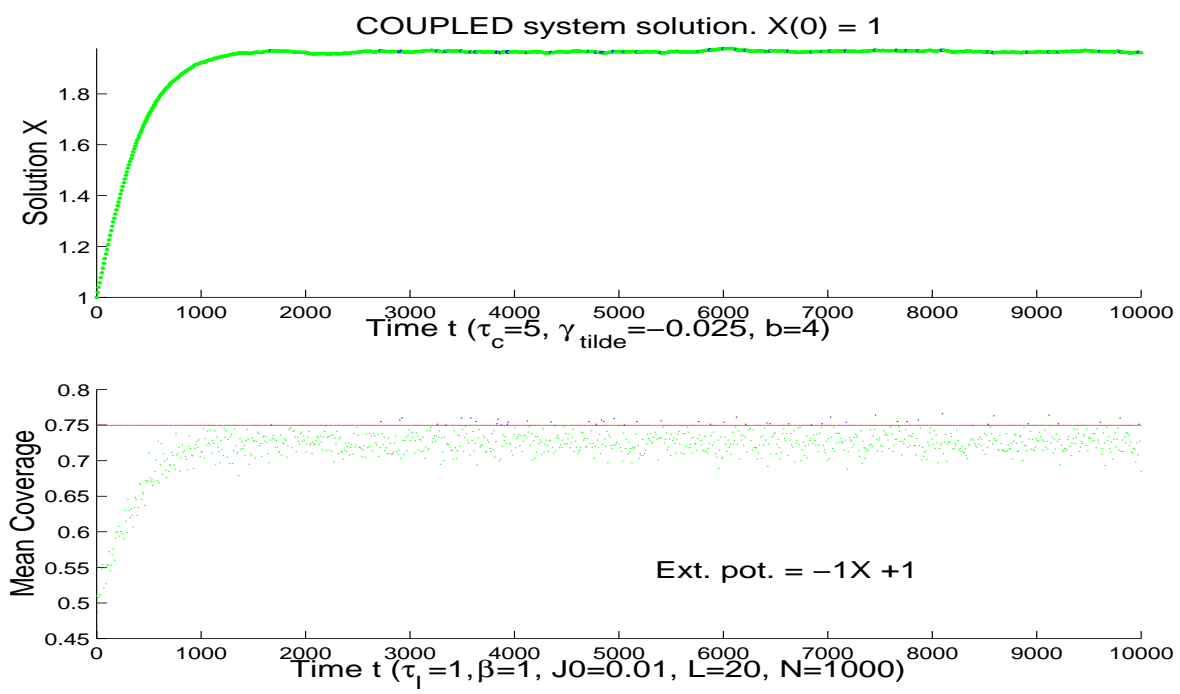

Fig. 4.1. Example 1. Monte Carlo simulated coupled system (4.2, 2.1) solution and mean coverage.

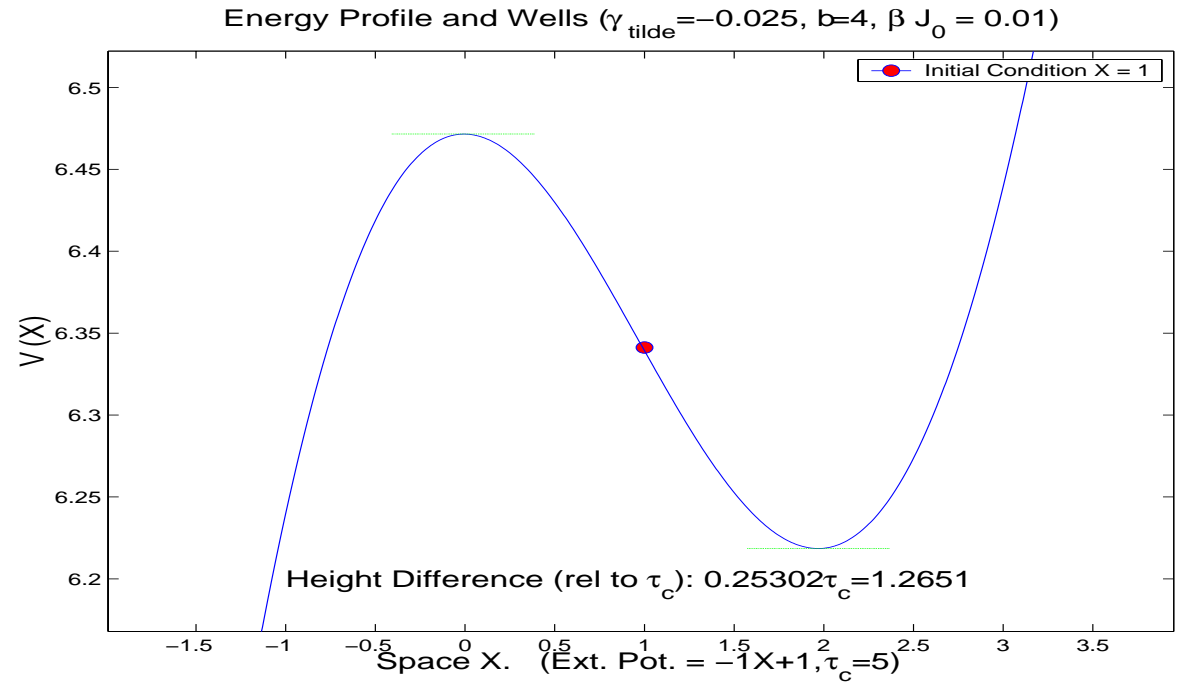

FIG. 4.2. Example 1. Energy profile for averaging principle system (4.3). The potential $V$ is understood through $-V^{\prime}=\bar{f}$. Parameters: $b=4, \tau_{c}=5$ and $\beta J_{0}=.01$ 

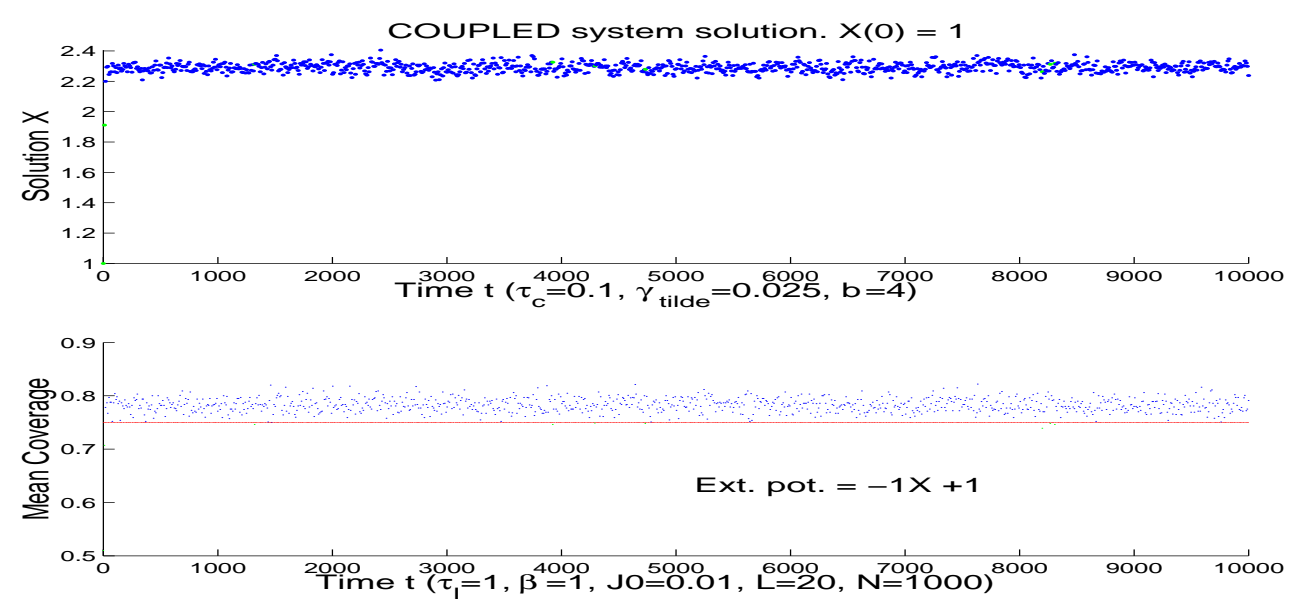

FiG. 4.3. Example 2. Monte Carlo simulated coupled system solution and mean coverage.

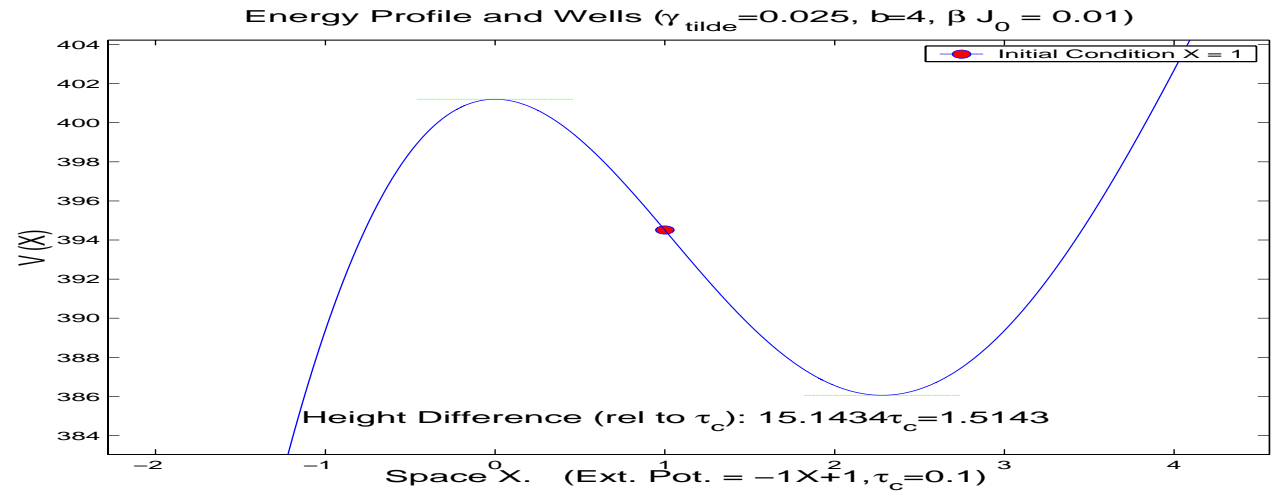

FIG. 4.4. Example 2. Energy profile corresponding to averaging principle system (4.3). The potential $V$ is understood through $-V^{\prime}=\bar{f}$. Parameters: $b=4, \tau_{c}=.1$ and $\beta J_{0}=.01$.

4.2. Saddle bifurcation. Contrary to the scalar bifurcation of the previous example - where we always have at least one fixed point - we now examine a saddle (fold) bifurcation example where two fixed points either appear or disappear. The specific dynamical system which we simulate is,

$$
\begin{cases}\frac{d X}{d t} & =r(\bar{\sigma})+\frac{\tilde{\gamma}}{\tau_{c}} X^{2}, \text { where we let } r(\bar{\sigma})=\frac{b}{\tau_{c}}(z-\bar{\sigma}) \\ \frac{d}{d t} \operatorname{Ef}(\sigma) & =E L f(\sigma, h(X)) \\ X(0) & =1 \quad \text { and random initial } \sigma, N=1000, L=20 .\end{cases}
$$

We apply the following type of external potential: $h(X)=c X+h_{0}$. As usual all parameters are provided at the tables which follow. The ODE in (4.4), depending on the sign of $r(\bar{\sigma})$ has the following stability profile: either 1 stable and 1 unstable node at: $\pm \sqrt{\frac{-r(\bar{\sigma}))}{\tilde{\gamma}}}$ or no nodes exist. The averaged ODE, (3.6) for the saddle bifurcation 
becomes,

$$
\left\{\begin{aligned}
\frac{d}{d t} \bar{x} & =\frac{b}{\tau_{c}}\left[z-u_{\beta, N}(h(\bar{x})]+\frac{\tilde{\gamma}}{\tau_{c}} \bar{x}^{2}\right. \\
\bar{x}_{0} & =x_{0} \equiv X(0)
\end{aligned}\right.
$$

Recall that following the discussion in Section 3.1, $u_{\beta, N}$ is approximated by the minimizer of (3.7).

We now present the comparisons for the complete Monte Carlo simulations of (4.4) against the solutions of (4.5) in Table 4.2. We apply a linear external potential of the form

$$
h(X)=c X+h_{0}
$$

with $c=5, h_{0}=-1$. Our parameters for this example are as follows: $\tau \equiv \tau_{c}=$ $.1,1,5, \beta J_{0}= \pm 2, .01, b=1, \tilde{\gamma}=-.05, z=.5$ and $\tau_{I}=1$. Note that the bifurcation occurs for $\bar{\sigma}=.5$.

The saddle example summarized in Table 4.2 is in a way typical in that it encompasses most of our findings between the Monte Carlo simulations and averaging principle predictions at once. We observe the following behavior between the two: agreement for the case of $\tau_{c}=5$ as expected, uncertain agreement for $\tau_{c}=1$ and last possible not agreement for $\tau_{c}=.1$. In fact for this case of $\tau_{c}=.1$ we clearly have substantial discrepancies (see Table 4.2).

There is a plausible explanation regarding some of the results and expected disagreements of solutions for the case of $\tau_{c}=.1$ to be found in Figures $4.5-4.10$. The relative height differences between the potential wells depicted in these figures are $.001,1.05$ and 1.11 for $\beta J_{0}=.01,2$ and -2 respectively. It is therefore not surprising for the case of $\beta J_{0}=.01$ and $\tau_{c}=.01$ that we observe the jump to the node at $-\infty$ (see Figure 4.11) instead of the averaging principle predicted solution of $X=.13$. Why are we not observing this "jump" for the remaining eight examples in that Table? First the jump does not occur for the remaining two cases of $\beta J_{0}=.01$ since as we can easily see in Figures 4.11 and 4.12 the noise level is reduced as we increase $\tau_{c}$ (see Table 5.1 for further comments on this behavior). The reason however that we do not observe this jump for the remaining six cases of $\beta J_{0}=2$ and -2 is mainly due to the increased depth (in relative heights) of their respective potential wells as can be seen in Figures 4.8 and 4.10 when compared to the potential well depicted in Figure 4.6.

However is this really the complete picture? We claim not! We therefore extend our (long equilibrated) simulations in time even further for the case of $\beta J_{0}=.01$ and $\tau_{c}=1$ in hope of observing a rare event - a jump from one potential well at .13 to the well at $-\infty$ given enough time. Indeed this is the case as can be seen in Figure 4.13 where such a jump is finally observed. Note that the choice of $\beta J_{0}=.01$ is important since the noise is greatest for this case and therefore we have better hope of observing such a jump in due time. In fact our claim is that given enough time we can observe a similar jump even for the case of $\tau_{c}=5$. Currently however, we have not observed this rare event (see Figure 4.14). See Section 5 for further comments and remarks regarding these observations. 
TABLE 4.5. Monte Carlo simulations versus averaging principle predictions for saddle bifurcation example. Note the remarkable agreement for the case of the averaging principle ODE regime $\tau_{c}=5$. Also note the expected not so good agreement for the opposite regime of $\tau_{c}=.1$

\begin{tabular}{|c|c|c|c|c|c|}
\hline \multirow{2}{*}{$\beta J_{0}$} & \multirow{2}{*}{$\begin{array}{l}\text { Char. } \\
\text { time }\end{array}$} & \multicolumn{4}{|c|}{ Monte Carlo Simulations Summary Statistics } \\
\hline & & & $\operatorname{StDev}(X)$ & $\overline{\bar{\sigma}}$ & $\operatorname{StDev}(\bar{\sigma})$ \\
\hline \multirow{3}{*}{.01} & $\tau=.1$ & $-\infty$ & - & .5 & .015 \\
\hline & $\tau=1$ & .1 & .062 & .5 & 015 \\
\hline & $\tau=5$ & .12 & 019 & .5 & 015 \\
\hline \multirow{3}{*}{2} & $\tau=.1$ & .1 & .032 & .5 & .022 \\
\hline & $\tau=1$ & .103 & .009 & .5 & 022 \\
\hline & $\tau=5$ & .103 & .003 & .5 & .021 \\
\hline \multirow{3}{*}{-2} & $\tau=.1$ & .3 & .017 & .5 & .01 \\
\hline & $\tau=1$ & .29 & .005 & .496 & .01 \\
\hline & $\tau=5$ & .29 & .002 & .49 & .01 \\
\hline
\end{tabular}

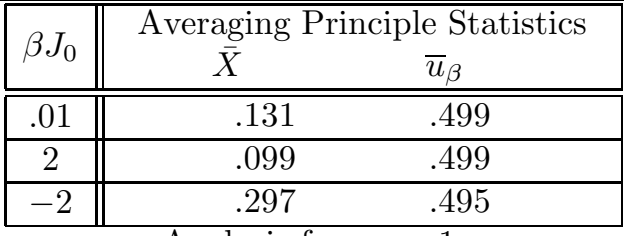

Analysis for $\tau_{c}=.1$ :

Case Characterization Aver. Princ. MC Simul. Rel. error

$\begin{array}{lllll}\beta J_{0}=.01 & \text { stable node at } & X_{A P}=0.13 & X_{M C}=-\infty & \infty \\ \beta J_{0}=2 . & \text { stable node at } & \bar{X}_{A P}=0.09 & \bar{X}_{M C}=0.1 & .1 \\ \beta J_{0}=-2 . & \text { stable node at } & \bar{X}_{A P}=0.29 & \bar{X}_{M C}=0.3 & .71\end{array}$

Analysis for $\tau_{c}=1$ :

Case Characterization Aver. Princ. MC Simul. Rel. error

\begin{tabular}{lllll}
\hline$\beta J_{0}=.01$ & stable node at & $X_{A P}=0.13$ & $X_{M C}=0.1$ & .87 \\
$\beta J_{0}=2$. & stable node at & $\bar{X}_{A P}=0.09$ & $\bar{X}_{M C}=0.103$ & .04 \\
$\beta J_{0}=-2$. & stable node at & $\bar{X}_{A P}=0.29$ & $\bar{X}_{M C}=0.29$ & 0
\end{tabular}

$\beta J_{0}=-2 . \quad$ stable node at $\quad \bar{X}_{A P}=0.29 \quad \bar{X}_{M C}=0.29 \quad 0$

Analysis for $\tau_{c}=5$ (Averaging Principle regime):

Case Characterization Aver. Princ. MC Simul. Rel. error

\begin{tabular}{llllc}
\hline$\beta J_{0}=.01$ & stable node at & $X_{A P}=0.13$ & $X_{M C}=0.12$ & .08 \\
$\beta J_{0}=2$. & stable node at & $\bar{X}_{A P}=0.09$ & $\bar{X}_{M C}=0.103$ & .04 \\
$\beta J_{0}=-2$. & stable node at & $\bar{X}_{A P}=0.29$ & $\bar{X}_{M C}=0.29$ & 0
\end{tabular}




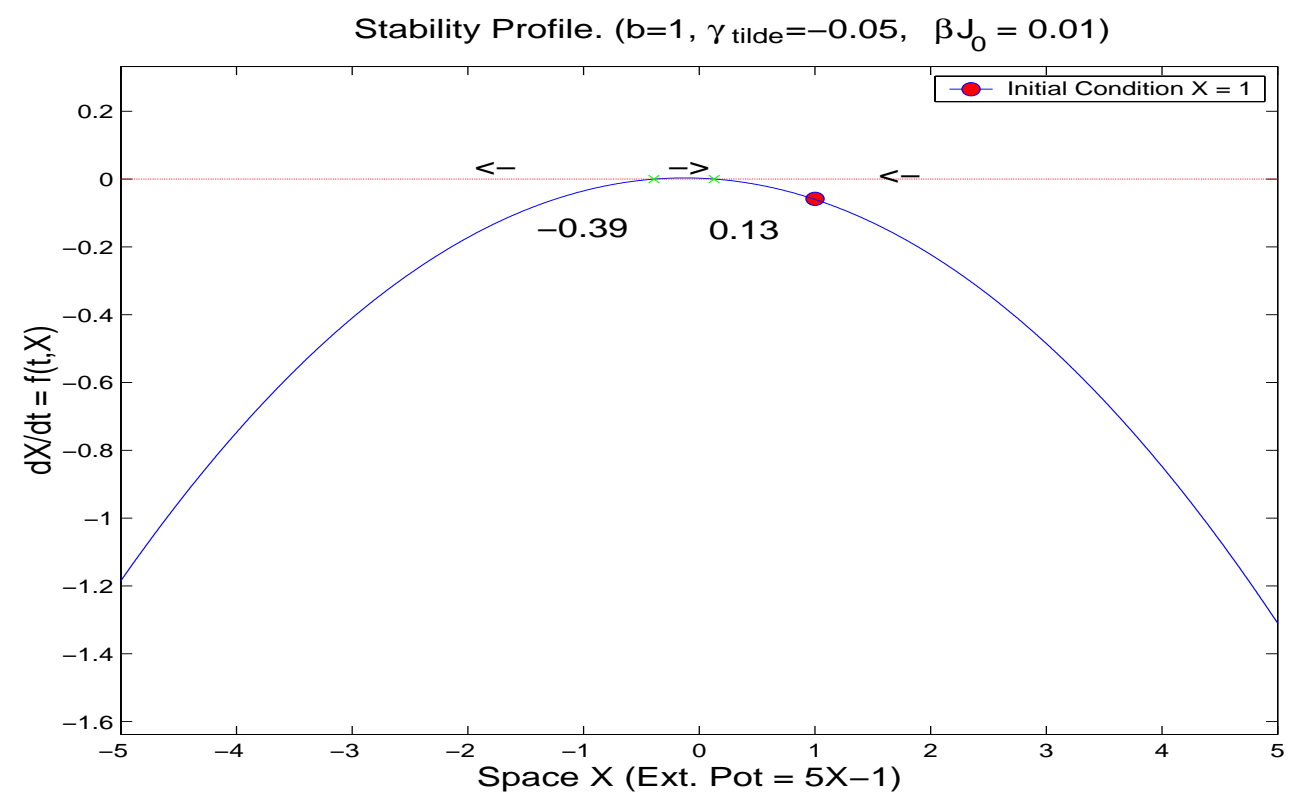

Fig. 4.5. Saddle ODE: Stability of (4.5). Parameters: $b=1 ., \tilde{\gamma}=-.05, \tau_{c}=1$ and $\beta J_{0}=.01$

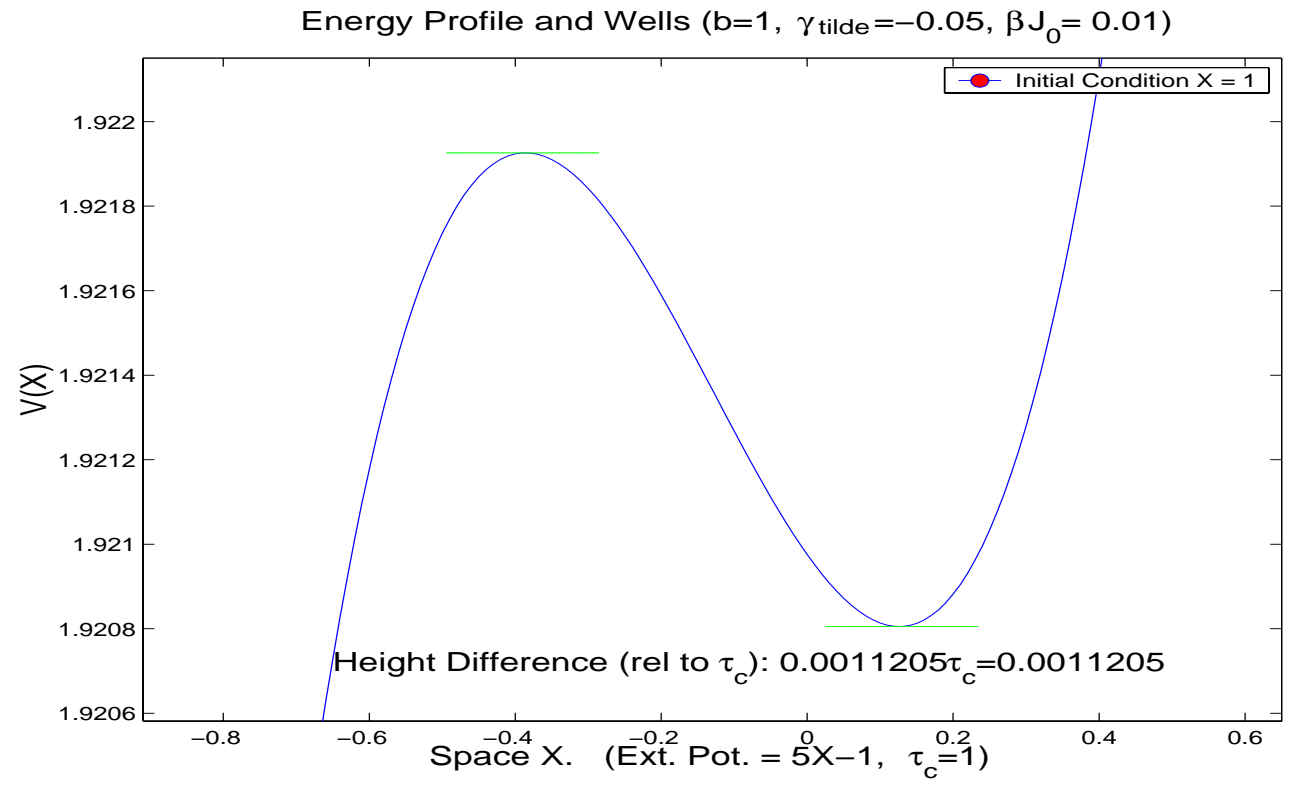

Fig. 4.6. Saddle ODE: Potential for (4.5). Parameters: $b=1 ., \tilde{\gamma}=-.05, \tau_{c}=1$ and $\beta J_{0}=.01$ 


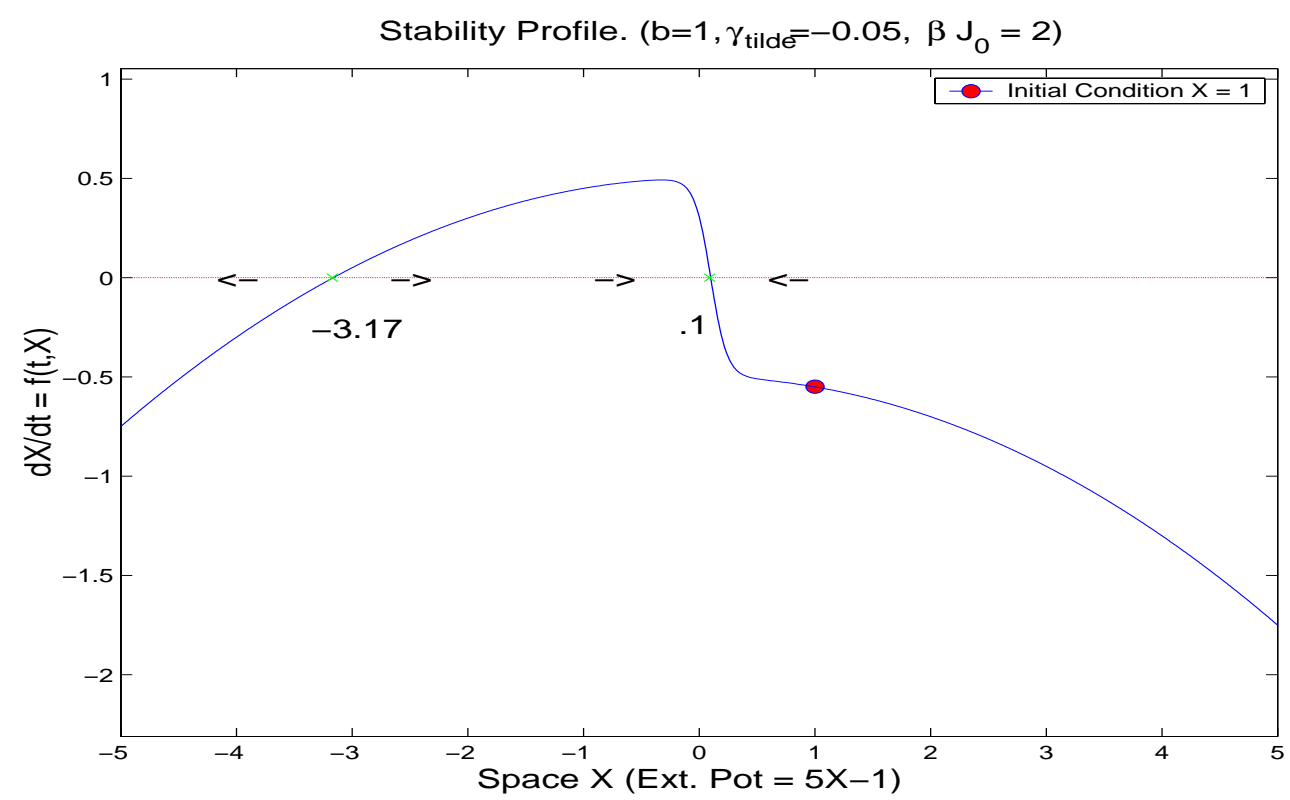

FIG. 4.7. Saddle ODE: Stability of (4.5). Parameters: $b=1 ., \tilde{\gamma}=-.05, \tau_{c}=1$ and $\beta J_{0}=2$.

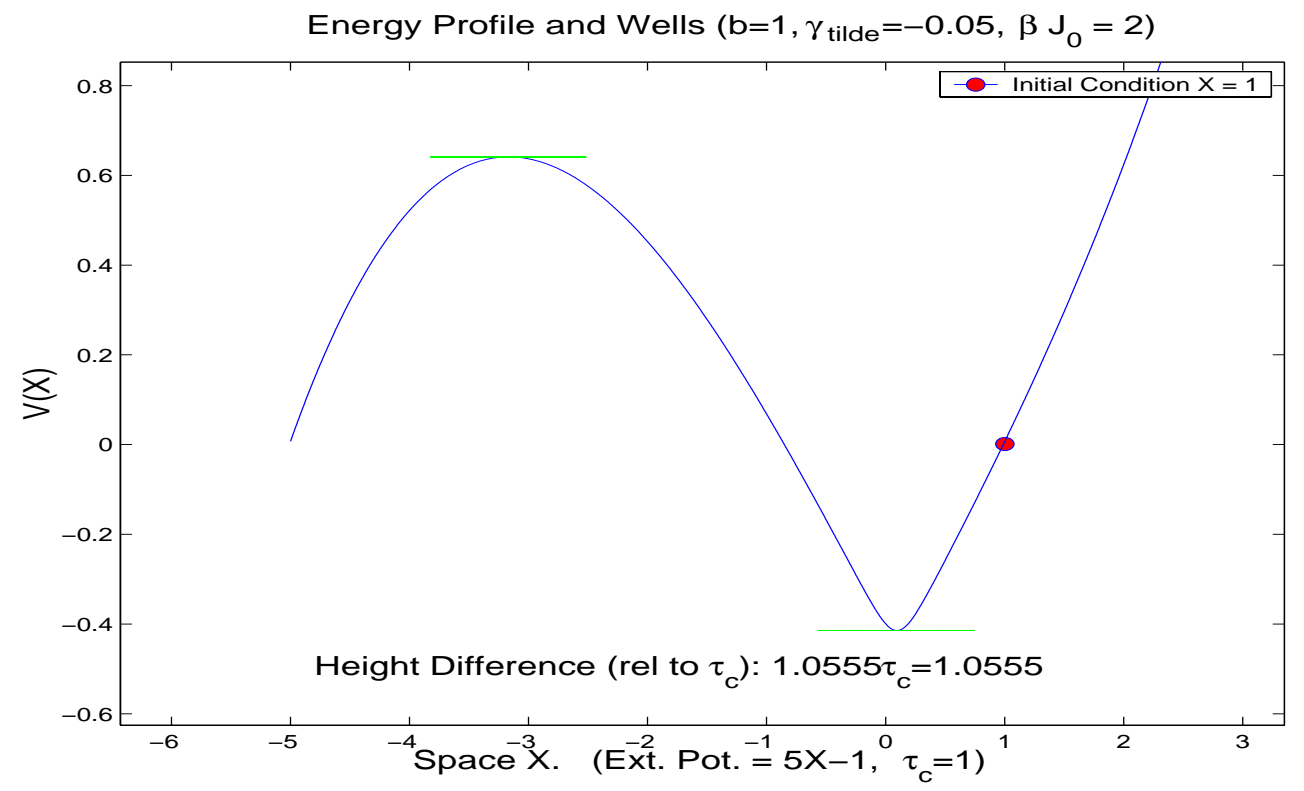

FIG. 4.8. Saddle ODE: Potential for (4.5). Parameters: $b=1 ., \tilde{\gamma}=-.05, \tau_{c}=1$ and $\beta J_{0}=2$. 


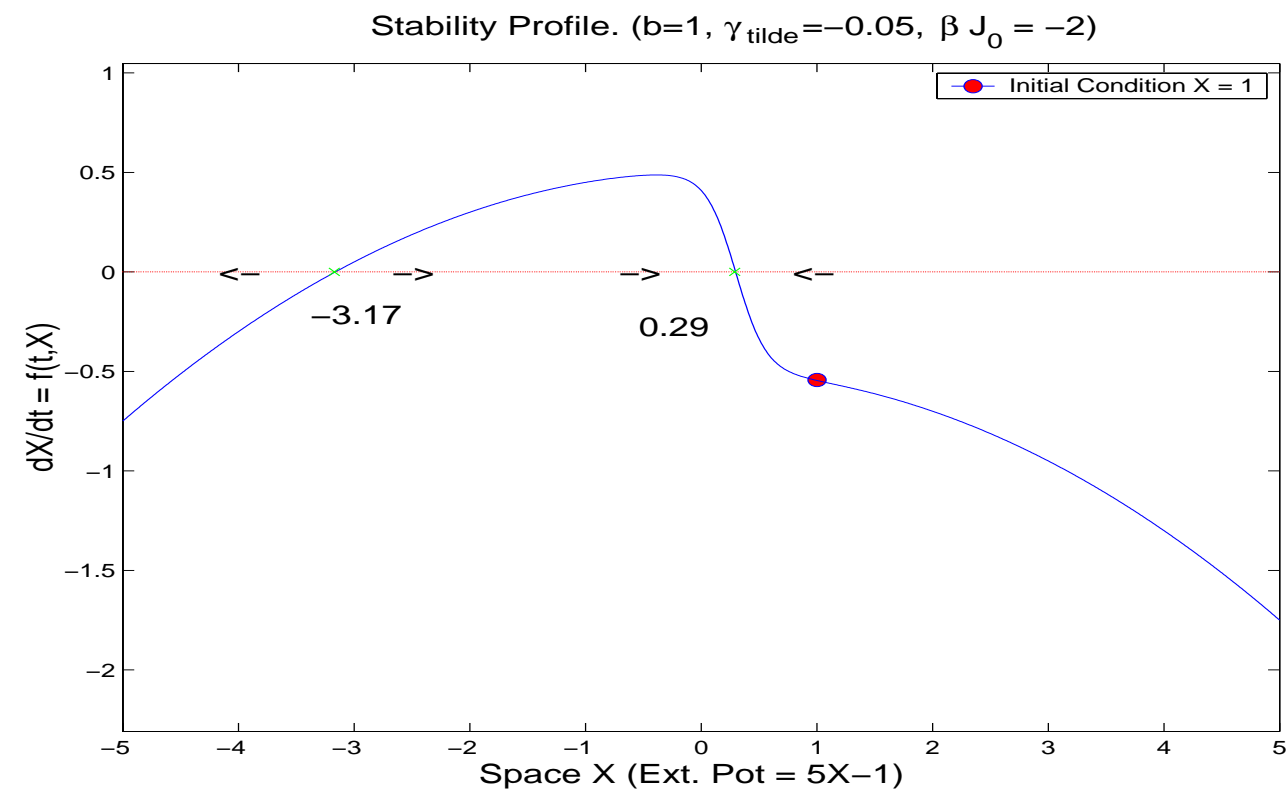

Fig. 4.9. Saddle ODE: Stability of (4.5). Parameters: $b=1 ., \tilde{\gamma}=-.05, \tau_{c}=1$. and $\beta J_{0}=-2$.

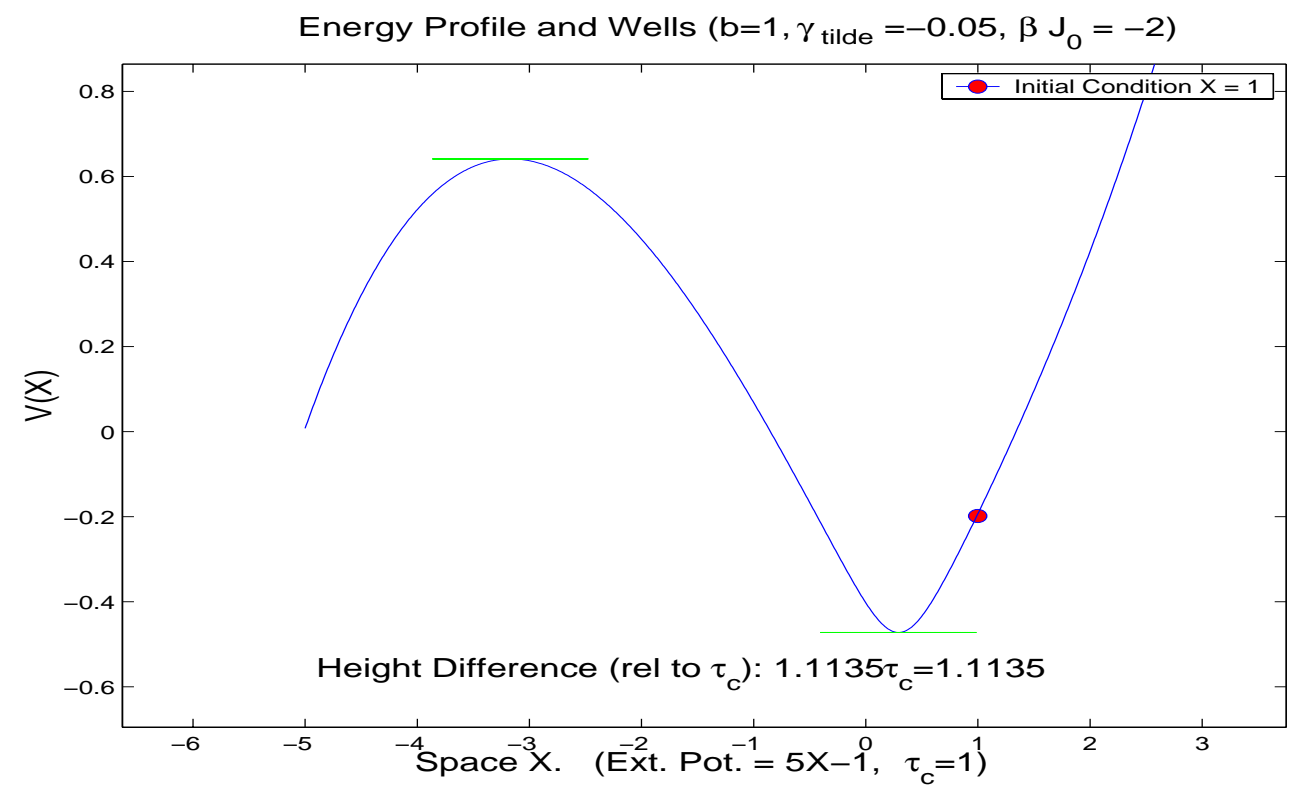

FIG. 4.10. Saddle ODE: Potential for (4.5). Parameters: $b=1 ., \tilde{\gamma}=-.05, \tau_{c}=1$. and $\beta J_{0}=-2$. 

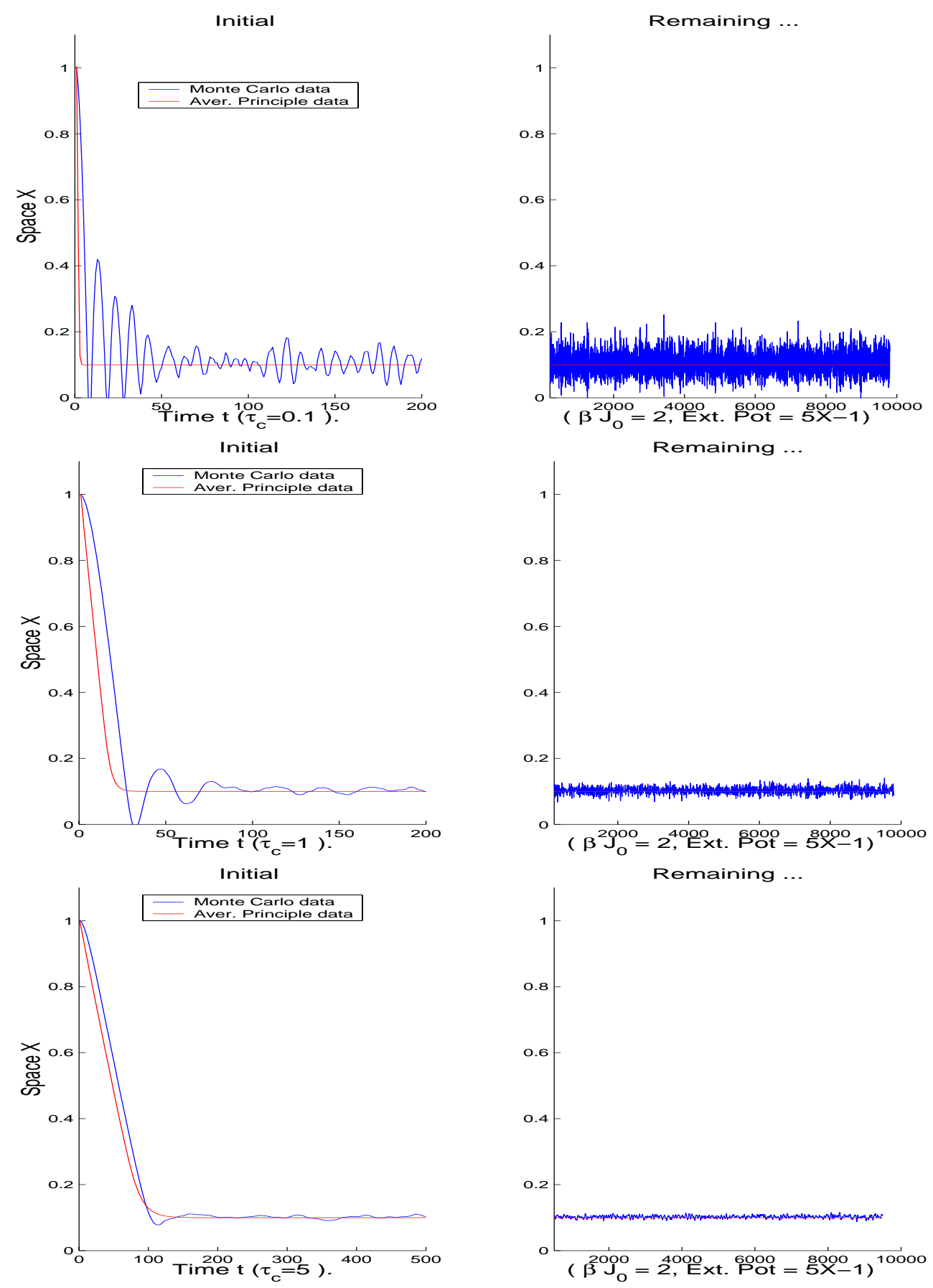

FIG. 4.11. Saddle ODE example: Solutions of both Monte Carlo and averaged ODE (4.5). Parameters: $b=1 ., \tilde{\gamma}=-.05, \beta J_{0}=2$ and $\tau_{c}=.1,1,5$ from top to bottom respectively. Another interesting point which becomes clear in these figures is the noise reduction with increasing $\tau_{c}$ (see also Table 5.1 regarding this observation). 
4.3. Hopf Bifurcation. For our last major example we examine the system comprised of the following Hopf bifurcation ODE and stochastic model:

$$
\left\{\begin{array}{rlrl}
\frac{d \vec{X}}{d t} & =\frac{1}{\tau_{c}}\left[A \vec{X}-|\vec{X}|^{2} \vec{X}\right], & \text { with } \vec{X}\left(t_{0}\right)=\vec{X}_{0}=\left(\begin{array}{c}
1 \\
1
\end{array}\right) \\
\frac{d}{d t} E f(\sigma) & =E L f(\sigma, h(X)), \quad N=1000, L=20 \quad \text { and random initial } \sigma .
\end{array}\right.
$$

where $A=\left(\begin{array}{cc}a(\bar{\sigma})+\gamma & -\omega \\ \omega & a(\bar{\sigma})-\gamma\end{array}\right),|\vec{X}|^{2}=X^{2}+Y^{2}$ and $a(\bar{\sigma})=b(\bar{\sigma}-z)$ with $b=4$ and $z=.5$. We apply a linear external potential

$$
h(\vec{X})=c\left(X+h_{0}\right)
$$

with $c=.5, h_{0}=-1$. and choose the remaining parameters as follows: $\omega=1, \tau_{I}=$ $1, \gamma=.9$. The stability behavior for this system depends on the sign of $a(\bar{\sigma})$ in the following manner,

- Stable node at $(X, Y)=(0,0)$ for $a(\bar{\sigma})<.5$.

- Limit cycle for $a(\bar{\sigma}) \geq .5$ with radius depending on the size of $\bar{\sigma}$ (nonsymmetric limit cycles occur for values of $\bar{\sigma}$ approaching 1 ).

We display in Figures 4.15-4.16 the stability behavior of the uncoupled Hopf ODE for the following fixed values of the noise parameter $\bar{\sigma}=.2, .5$ and .9 .

The equivalent averaging principle equation (3.6) becomes,

$$
\left\{\begin{aligned}
\frac{d}{d t} \bar{x}_{t} & =\frac{1}{\tau_{c}}\left[\left(a\left(u_{\beta, N}\left(h\left(\bar{x}_{t}\right)\right)\right)+\gamma\right) \bar{x}_{t}-\omega \bar{y}_{t}-\bar{x}_{t}^{3}-\bar{x}_{t}^{2} \bar{y}_{t}\right] \\
\frac{d}{d t} \bar{y}_{t} & =\frac{1}{\tau_{c}}\left[\omega \bar{x}_{t}+\left(a\left(u_{\beta, N}\left(h\left(\bar{x}_{t}\right)\right)\right)-\gamma\right) \bar{y}_{t}-\bar{x}_{t}^{2} \bar{y}_{t}-\bar{y}_{t}^{3}\right] \\
\bar{x}_{0} & =\bar{x}_{0}
\end{aligned}\right.
$$

where all parameters $a, \omega, \gamma, \vec{X}_{0}$ as provided already. Recall that following the discussion in Section 3.1, $u_{\beta, N}$ is approximated by the minimizer of (3.7). We examine the agreement between (4.6) and (4.7) under the following cases of $\tau_{c}=.1,1,5$ and $\beta J_{0}= \pm 2, .01$.

In Tables 4.3, 4.3 and 4.3 we present comparisons of both the numerical Monte Carlo solutions of (4.6) and averaging principle solutions of (4.7). Although representing averages in these tables is a very crude comparison it still provides one more indicator of the similarities or differences between the stochastic model and the averaging principle system. Notice also that in Table 4.3 comparisons include the cases of $\tau_{c}=50$ and 20 so as to further emphasize the range of validity of the averaging principle theory.

As expected there is remarkable agreement for the case of the averaging principle regime $\tau_{c}=5$ (or higher) which for example can be seen in Figure 4.17. Notice however the progressive deterioration in agreement in the comparisons depicted in Figures $4.17,4.21$ and 4.22 as $\tau_{c}$ varies through 5,1 and .1 respectively. The structure of the solutions becomes clear when we look at their respective orbits and probability density functions in Figures 4.18 and 4.23. Further information about the structure of these solutions can be obtained by studying the long time averages in Figures 4.19 and 4.20 and correlations in Figures 4.24 and 4.25. Additional comments are found in the discussion Session 5. 
TABLE 4.6. Monte Carlo versus averaging principle. Although this is a very crude comparison it is displayed here as another indicator of the similarities or differences between the stochastic model and the averaging principle system. This is a Hopf bifurcation example for $\beta J_{0}=-2$.

\begin{tabular}{|c|c||cc|cc|cc|}
\hline $\begin{array}{c}\text { Char. } \\
\text { time }\end{array}$ & & $\bar{X}$ & $\operatorname{StDev}(X)$ & $\bar{Y}$ & $\operatorname{StDev}(Y)$ & $\begin{array}{c}\bar{\sigma} \\
\bar{u}_{\beta}\end{array}$ & $\begin{array}{c}\operatorname{StDev}(\bar{\sigma}) \\
\operatorname{StDev}\left(\bar{u}_{\beta}\right)\end{array}$ \\
\hline \hline$\tau=5$ & MC: & -.0019 & .11 & 0.011 & 0.11 & .50 & .025 \\
& AP: & -.0008 & .07 & 0.005 & 0.07 & .50 & .014 \\
\hline$\tau=1$ & MC: & -.011 & .19 & 0.031 & 0.18 & .50 & .034 \\
& AP: & -.015 & .17 & 0.033 & 0.16 & .50 & .033 \\
\hline$\tau=.1$ & MC: & -.054 & .54 & -0.08 & 0.33 & .49 & .047 \\
& AP: & -.21 & .60 & -0.18 & 0.37 & .53 & .11 \\
\hline
\end{tabular}

TABLE 4.7. Monte Carlo versus averaging principle. Although this is a very crude comparison it is displayed here as another indicator of the similarities or differences between the stochastic model and the averaging principle system. This is a Hopf bifurcation example for $\beta J_{0}=2$

\begin{tabular}{|c|c||cc|cc|cc|}
\hline $\begin{array}{c}\text { Char. } \\
\text { time }\end{array}$ & & $\bar{X}$ & $\operatorname{StDev}(X)$ & $\bar{Y}$ & $\operatorname{StDev}(Y)$ & $\begin{array}{c}\bar{\sigma} \\
\bar{u}_{\beta}\end{array}$ & $\begin{array}{c}\operatorname{StDev}(\bar{\sigma}) \\
\operatorname{StDev}\left(\bar{u}_{\beta}\right)\end{array}$ \\
\hline \hline$\tau=50$ & MC: & -.030 & .95 & -.036 & .86 & .914 & .071 \\
& AP: & -.088 & .97 & -.068 & .87 & .92 & .075 \\
\hline$\tau=20$ & MC: & -.01 & .95 & -0.02 & 0.88 & .91 & .088 \\
& AP: & -.07 & .97 & -0.05 & 0.87 & .92 & .073 \\
\hline$\tau=5$ & MC: & -.01 & .95 & -0.02 & 0.86 & .91 & .071 \\
& AP: & -.07 & .97 & -0.05 & 0.87 & .92 & .075 \\
\hline$\tau=1$ & MC: & -.02 & .97 & -0.03 & 0.85 & .91 & .065 \\
& AP: & -.07 & .98 & -0.05 & 0.87 & .92 & .076 \\
\hline$\tau=.1$ & MC: & -.03 & 1.05 & -0.05 & 0.85 & .91 & .027 \\
& AP: & -.15 & 1.04 & -0.09 & 0.88 & .92 & .081 \\
\hline
\end{tabular}

TABLE 4.8. Monte Carlo versus averaging principle. Although this is a very crude comparison it is displayed here as another indicator of the similarities or differences between the stochastic model and the averaging principle system. This is a Hopf bifurcation example for $\beta J_{0}=.01$

\begin{tabular}{|c|c||cc|cc|cc|}
\hline $\begin{array}{c}\text { Char. } \\
\text { time }\end{array}$ & & $\bar{X}$ & $\operatorname{StDev}(X)$ & $\bar{Y}$ & $\operatorname{StDev}(Y)$ & $\begin{array}{c}\overline{\bar{\sigma}} \\
\bar{u}_{\beta}\end{array}$ & $\begin{array}{c}\operatorname{StDev}(\bar{\sigma}) \\
\operatorname{StDev}\left(\bar{u}_{\beta}\right)\end{array}$ \\
\hline \hline$\tau=5$ & MC: & -.0002 & .10 & -.0004 & 0.10 & .50 & .01 \\
& AP: & -.0002 & .09 & -.0003 & 0.09 & .50 & 0.00 \\
\hline$\tau=1$ & MC: & 0.0 & .18 & 0.0 & 0.16 & .50 & .01 \\
& AP: & -.0001 & .17 & 0.0001 & 0.15 & .50 & 0.0 \\
\hline$\tau=.1$ & MC & .0003 & .56 & 0.0 & .35 & .50 & .015 \\
& AP & -.0013 & .56 & .0011 & .34 & .50 & 0.0 \\
\hline
\end{tabular}



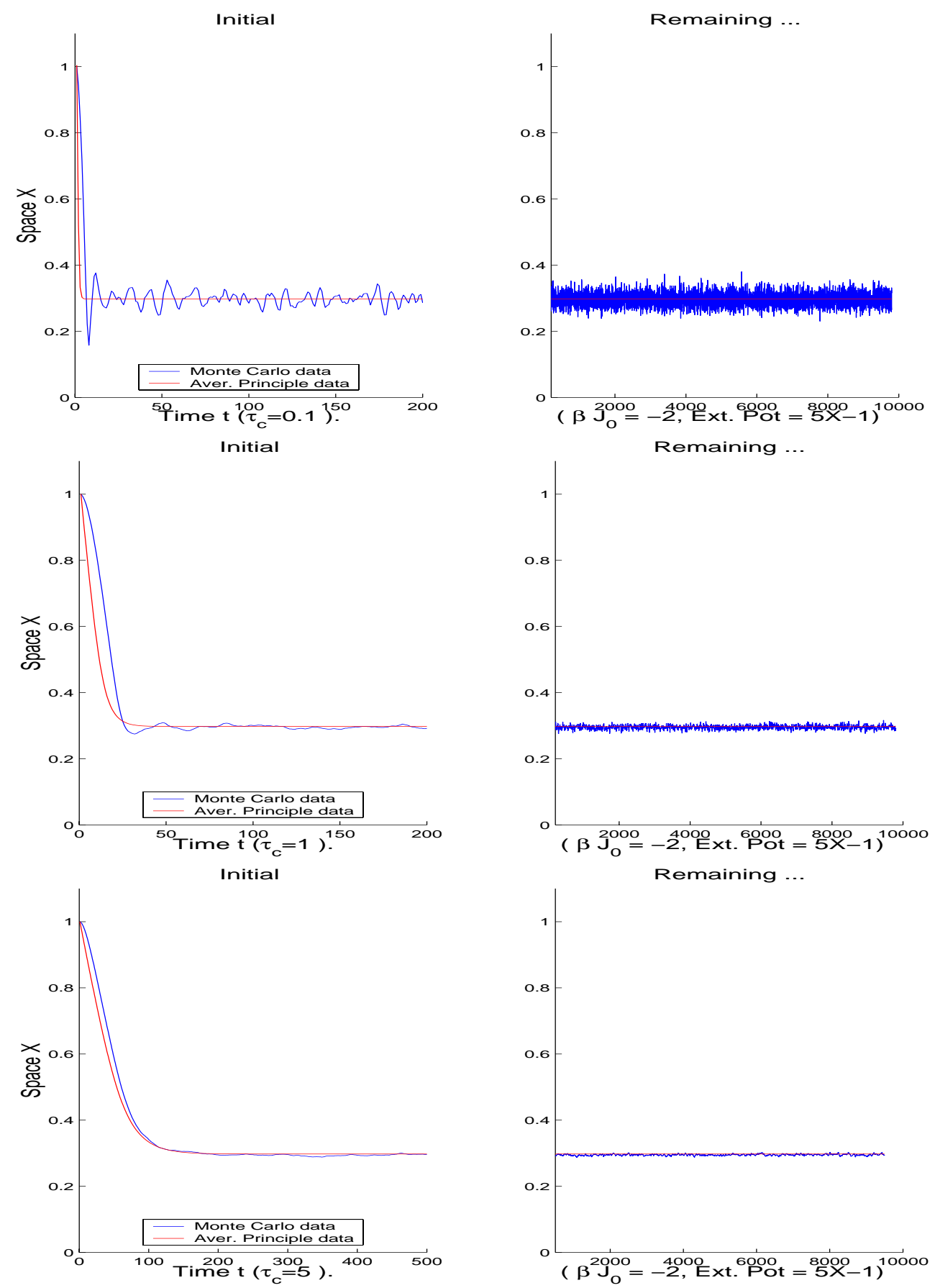

FIG. 4.12. Saddle ODE example: Solutions of both Monte Carlo and averaged ODE (4.5). Parameters: $b=1 ., \tilde{\gamma}=-.05, \beta J_{0}=-2$ and $\tau_{c}=.1,1,5$ from top to bottom respectively. Once again notice the noise reduction with increasing $\tau_{c}$ (see also Table 5.1 regarding this observation). 

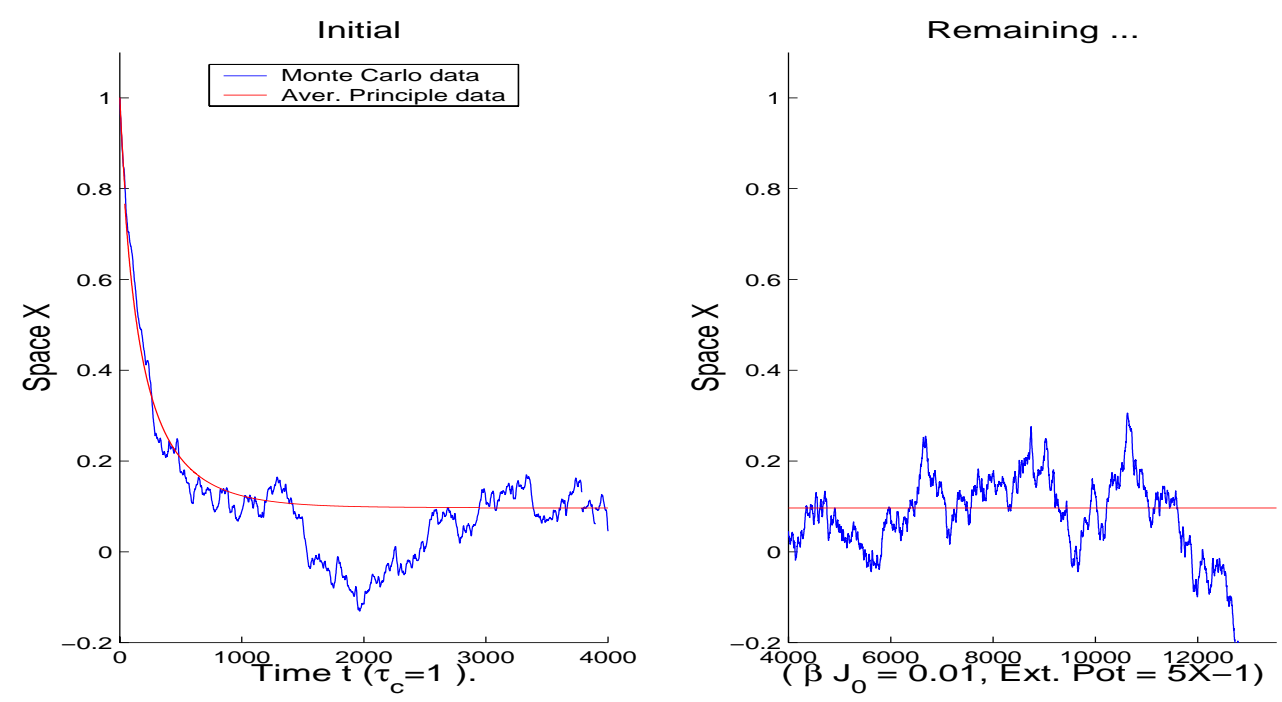

FIG. 4.13. Saddle ODE example: Extended time runs. Solutions of both Monte Carlo and reduced averaged ODE. Parameters: $b=1 ., \tilde{\gamma}=-.05, \tau_{c}=1$ and $\beta J_{0}=.01$. Note the jump attained by the system to the node at $-\infty$. The size of noise is substantial so that given enough time we observe the rare event.
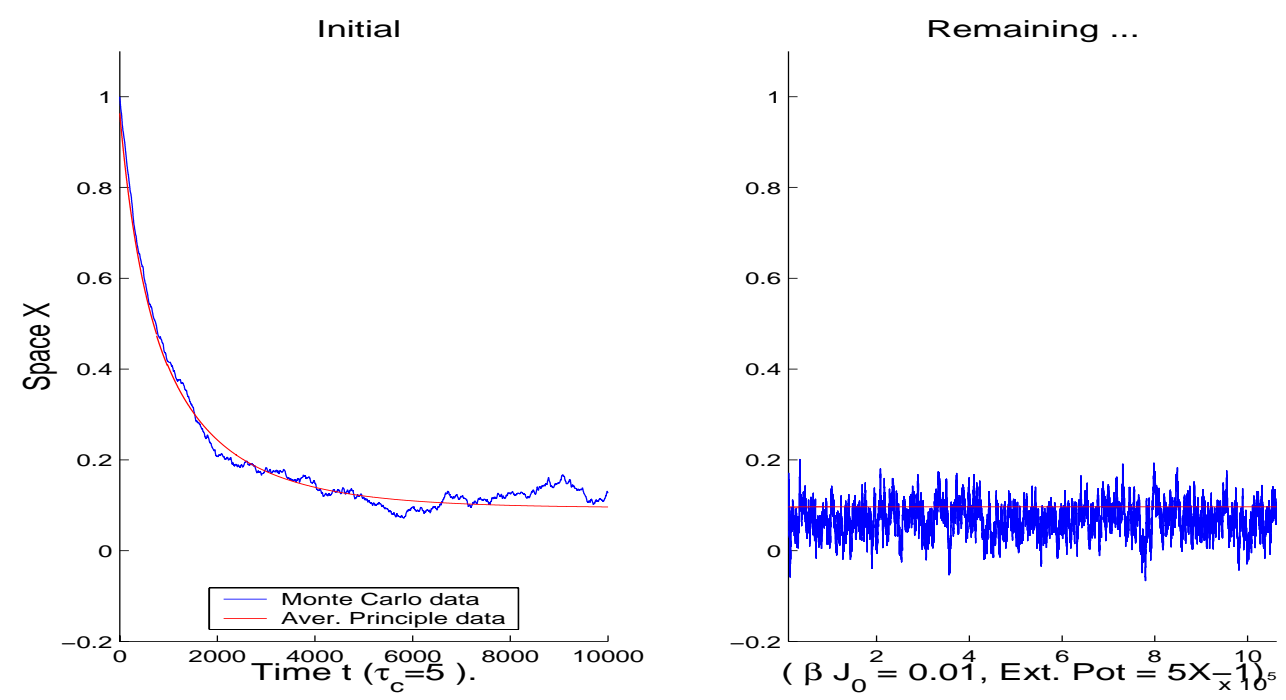

FIG. 4.14. Saddle ODE example: Extended time runs. Solutions of both Monte Carlo and reduced averaged ODE. Parameters: $b=1 ., \tilde{\gamma}=-.05, \tau_{c}=5$ and $\beta J_{0}=.01$. We have run further this simulation (not presented here) but even for very long runs we have not observed the rare event although it is expected theoretically. It is important to note that the noise is less than the case of $\tau_{c}=1$ and this creates an added hurdle in order to observe the jump to the node at $-\infty$. 


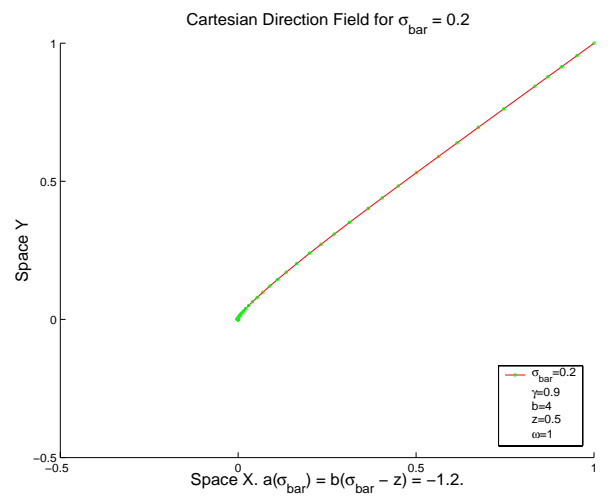

FIG. 4.15. Direction field for $\bar{\sigma}=.2$
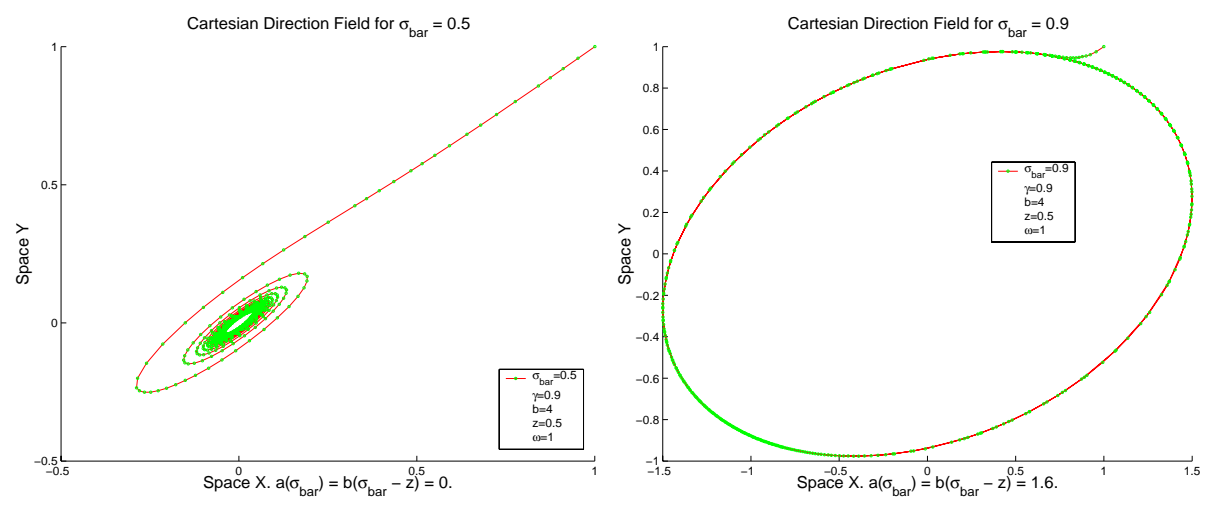

FIG. 4.16. Direction fields for fixed $\bar{\sigma}=.5$ and .9 respectively.
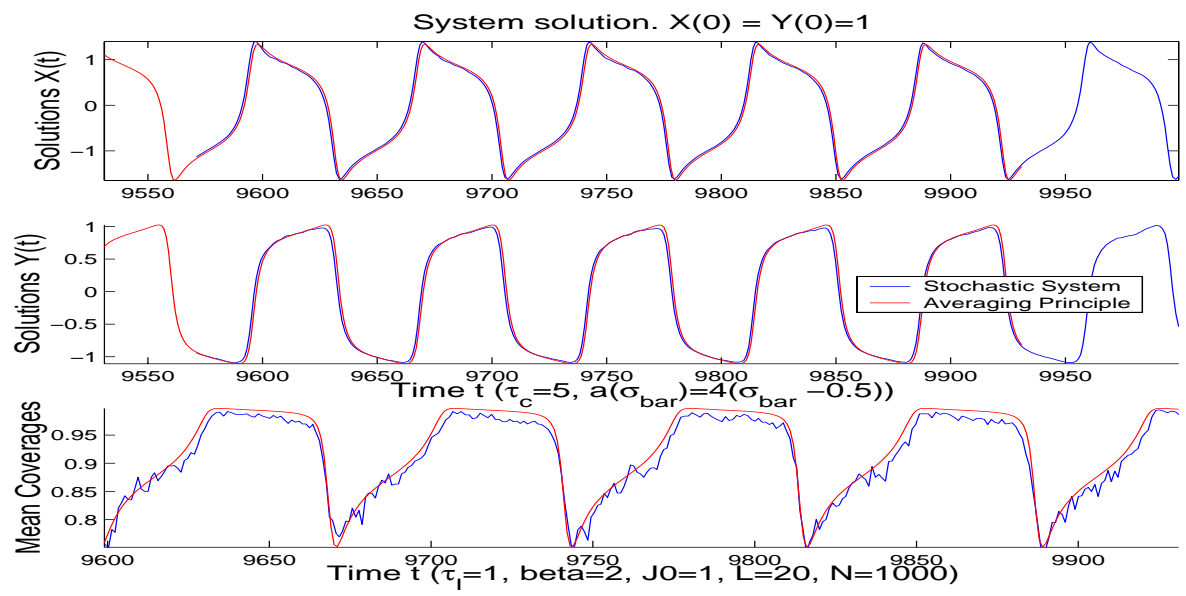

FIG. 4.17. Hopf ODE case: Comparisons between the solutions of the averaged ODE (4.7) and the coupled system (4.6). Parameters $\tau_{c}=5$ and $\beta J_{0}=2$. 

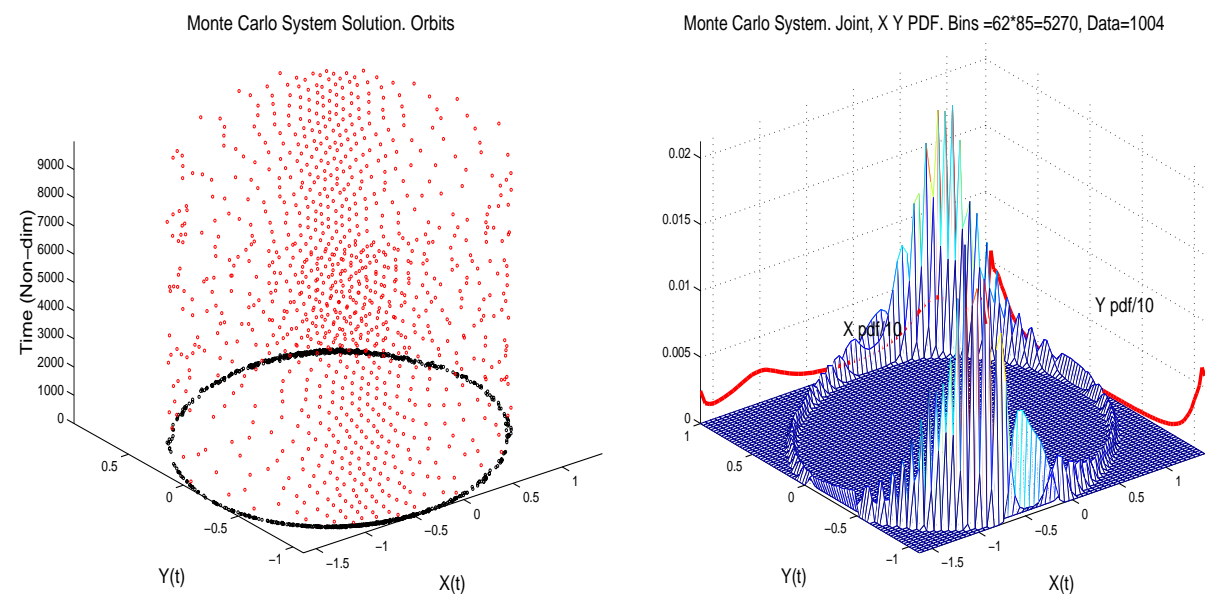

FIG. 4.18. Hopf ODE case: Monte Carlo system (4.6) orbits and PDFs. Parameters: $\tau_{c}=5$ and $\beta J_{0}=2$.
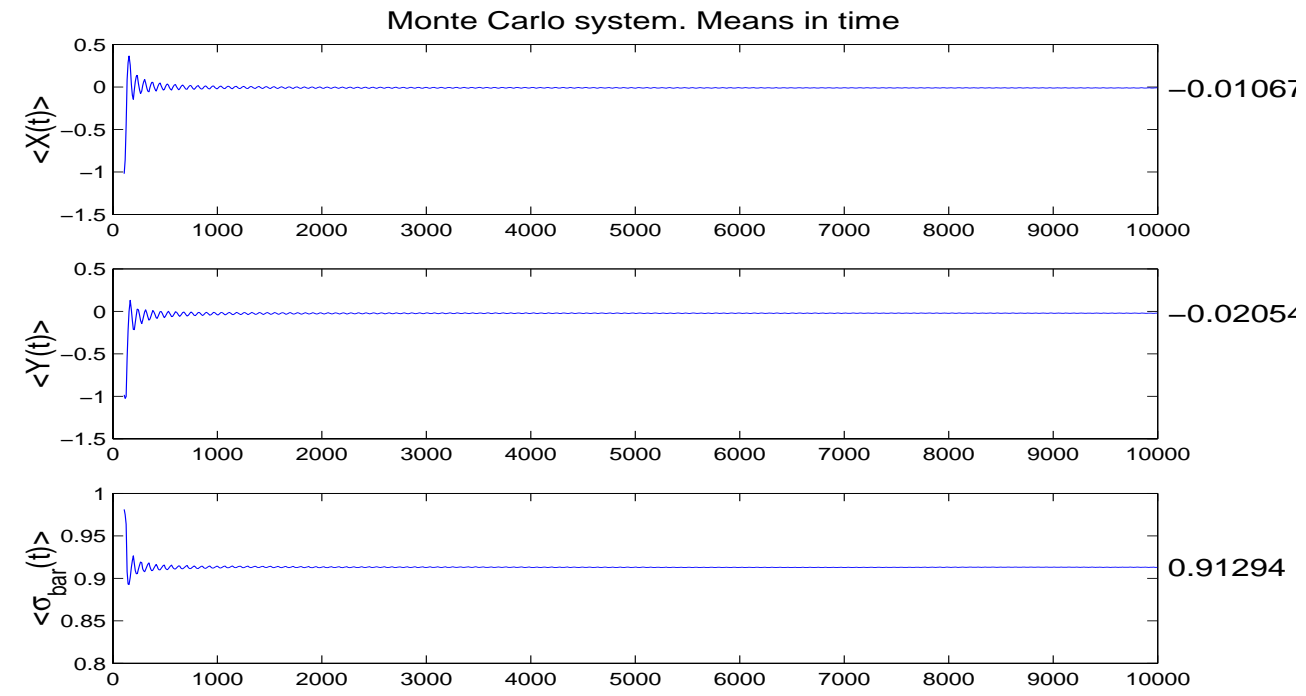

FIG. 4.19. Hopf ODE case: Monte Carlo system (4.6) averages in time. Parameters: $\tau_{c}=5$ and $\beta J_{0}=2$. 

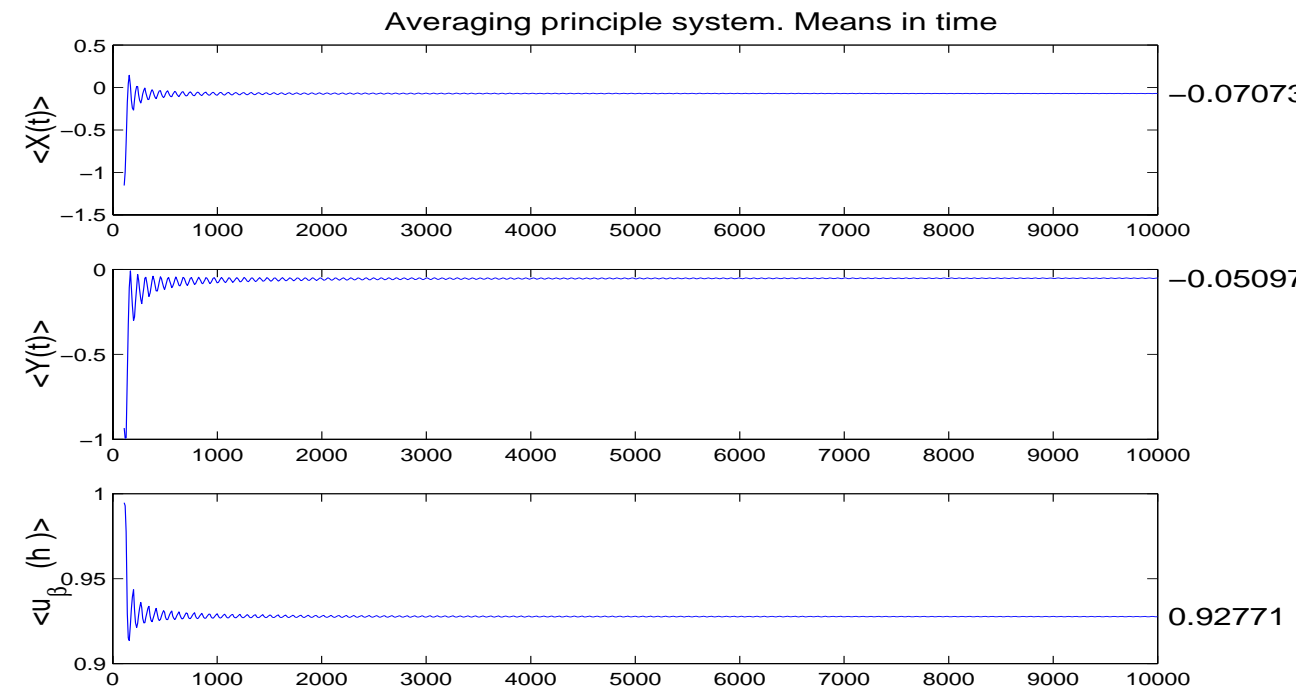

Fig. 4.20. Hopf ODE case: Eq. (4.7) averages in time. Parameters: $\tau_{c}=5$ and $\beta J_{0}=2$.
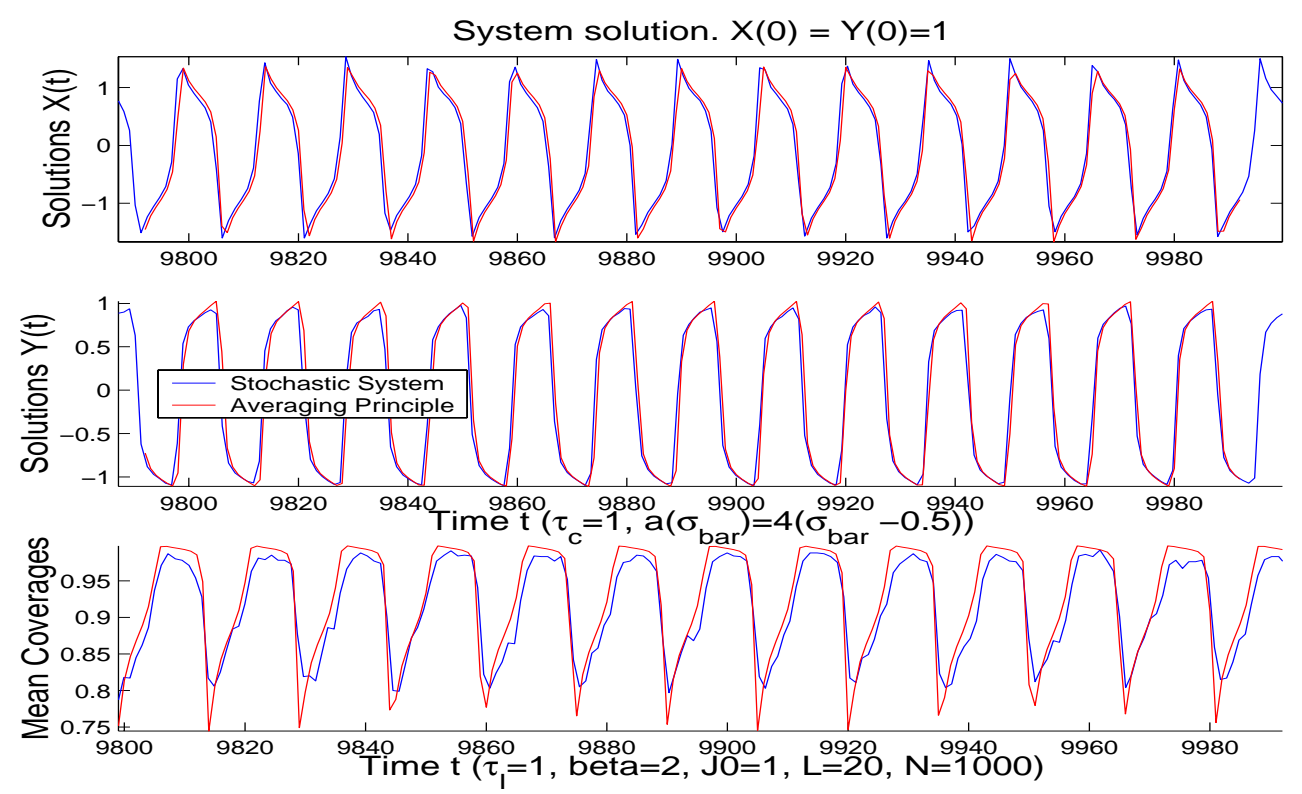

FIG. 4.21. Hopf ODE case: Comparisons between the solutions of the averaged ODE (4.7) and the coupled system (4.6). Parameters $\tau_{c}=1$ and $\beta J_{0}=2$. 

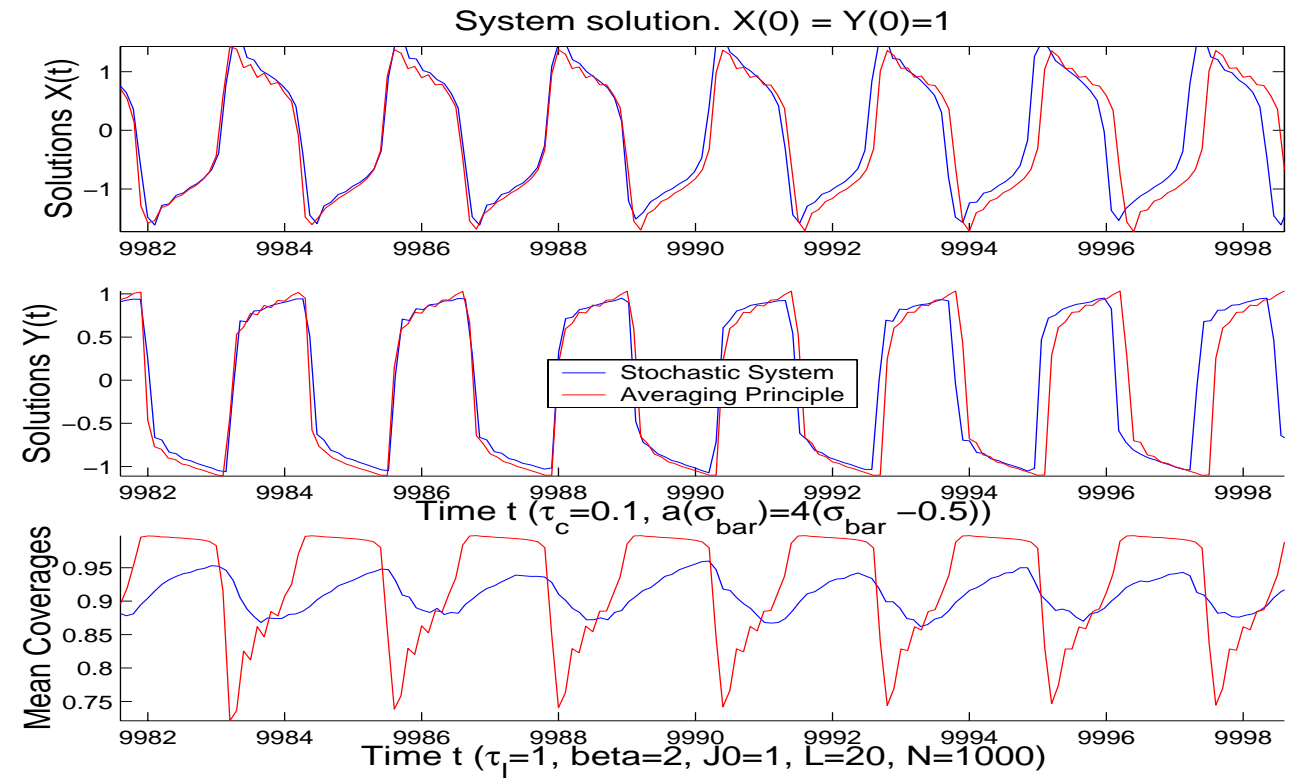

FIG. 4.22. Hopf ODE case: Comparisons between the solutions of the averaged ODE (4.7) and the coupled system (4.6). Parameters $\tau_{c}=.1$ and $\beta J_{0}=2$.

\section{Discussion of the results}

We presented a number of numerical comparisons of Monte Carlo simulations of the full coupled system (2.1), (2.2) against the numerical solution of the reduced ODE (4.3) as obtained by averaging principle theory (for $\tau=\tau_{c} / \tau_{I} \rightarrow \infty$ ). However we also include examples which are not in the range of $\tau>>1$ so as to further understand the extent of validity of the theoretical predictions. Our simulations were designed to correspond to the regime where phase transitions do not occur.

(A) Brief analysis of scalar results: in the two numerical examples shown (and several others not presented here) which were compared with the averaging principle theory predictions we have observed the following behavior:

-Overall agreement of Monte Carlo simulations with the reduced averaged equation; however in other simulations not presented here we have also established disagreement for the cases of $\tau_{c}=.1$ as we would expect.

(B) Brief analysis of saddle results: we observe complete agreement between the Monte Carlo simulations and the averaging principle predictions for the range of validity of the averaging principle theory and non-agreement otherwise.

- The averaged solution saddle bifurcation converges to finite stable nodes which are in complete agreement for both the Monte Carlo and averaging principle systems for the case of $\tau_{c}>>1$.

-Non-agreement for the case of $\tau=.1$ and 1 (see Table 4.2 and Figure 4.13).

Naturally, we observe the greatest noise for the case of $\tau_{c}=.1$ (see Figures 4.11 and 4.12, Table 5.1 and Remark 5.2). It is remarkable that with the noise induced in this case, the Monte Carlo simulation can "jump" over potential wells of the ODE and reach the other nodes (something not possible for a deterministic solution). Overall this result is not surprising however since, once again, the case of $\tau_{c}=.1$ is beyond the validity of the averaging principle theory $\left(\tau_{c}>>\tau_{I}\right)$ as was established in Section 

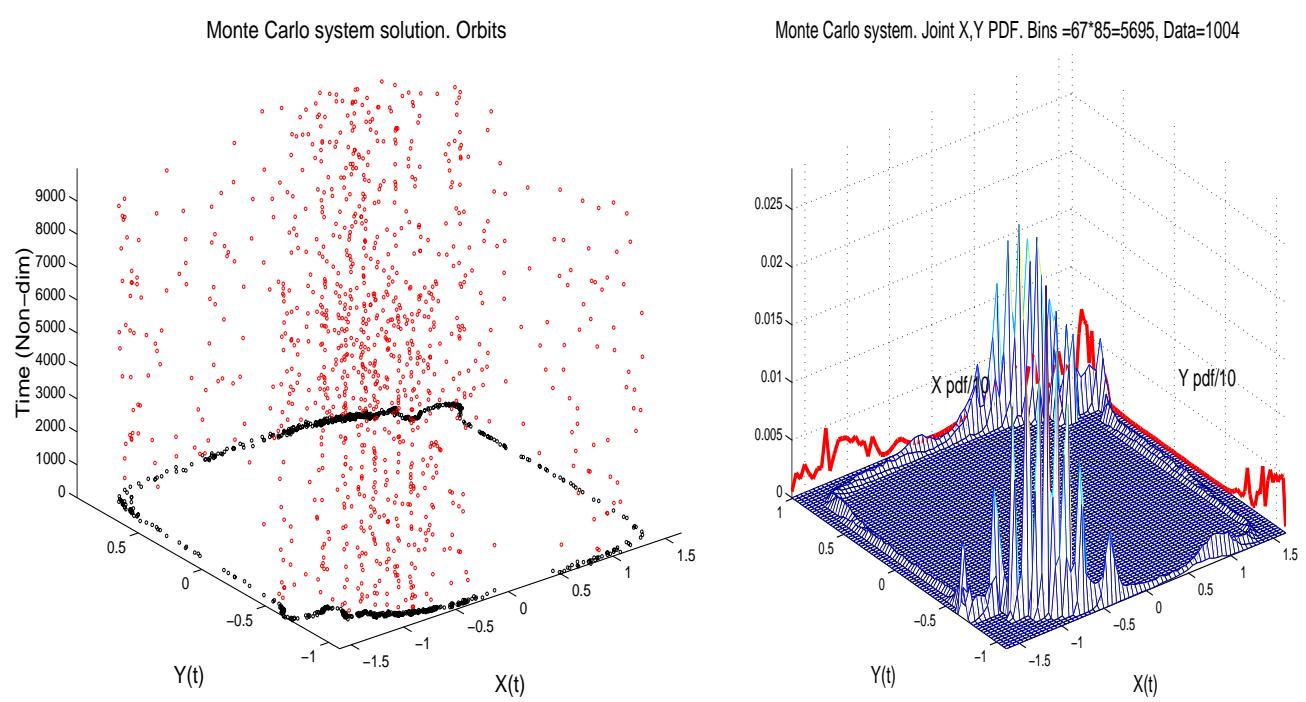

FIG. 4.23. Hopf ODE case: Monte Carlo system orbits and PDFs. Parameters: $\tau_{c}=.1$ and $\beta J_{0}=2$.

3. In this case rare events drive the system out of a stable node and into another such node, given enough time. This however is not, and could not possibly be, captured by the averaging principle system (4.5).

(C) Brief Analysis of Hopf Bifurcation Results: the Hopf bifurcation ODE display convergence to either the stable node at $(0,0)$ or a limit cycle. As expected the cases of low values for $\tau_{c}=.1$ are less in agreement with the averaging principle solutions (see Figure 4.22). Therefore we have once again the expected disagreement due to low $\tau_{c}$ values or high noise. Otherwise we observe remarkable agreement with the averaged equation.

REMARK 5.1. At times there is a slight but consistent error between the averaging principle and Monte Carlo system solutions (the averaging principle solution appears slightly higher than the Monte Carlo simulation). This is an expected artifact however of the fact that we approximate $u_{\beta, N}(h)$ through $u_{\beta}(h)$ from (3.8).

REMARK 5.2. It is important to underline here the effect of noise on the parameters that influence the simulations and how exactly this influence is manifested.

- Naturally, noise is influenced via the value of $\beta J_{0}$ since $\beta$ signifies inverse temperature and therefore we would expect that as,

$$
\beta \rightarrow \infty \text { the noise decreases. }
$$

- Similarly we know that the number of lattice nodes affects noise since the results are always averaged before presented (or used in the ODE). In that respect,

$$
\text { noise decreases as } N \rightarrow \infty \text {. }
$$

- Last, the characteristic times $\tau_{c}$ and $\tau_{I}$ also affect noise. We can see this effect by considering limiting situations. For instance assume that $\tau_{I} \rightarrow 0$ which would imply that the stochastic equilibrates immediately. As such the 
Monte Carlo system. Correlations in time Lag
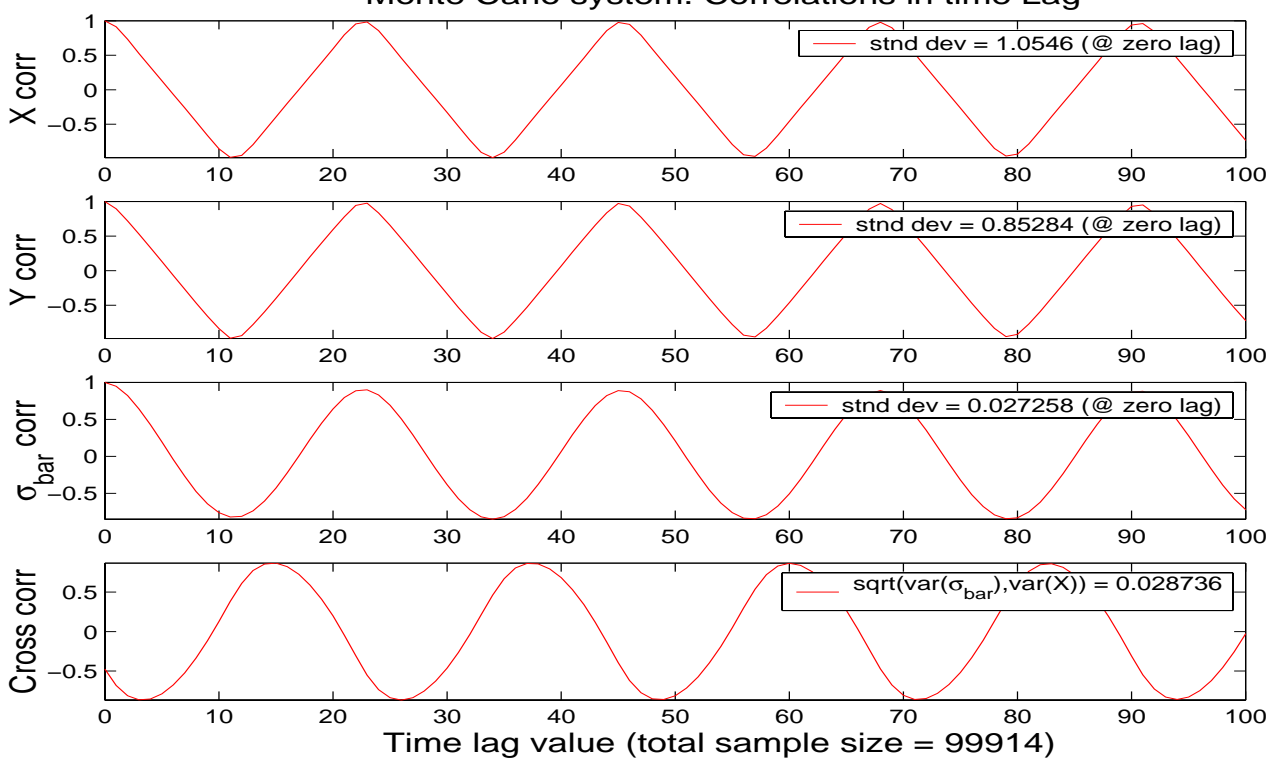

FIG. 4.24. Hopf ODE case: Monte Carlo system correlations in time lag. Parameters: $\tau_{c}=.1$ and $\beta J_{0}=2$. (Cross correlations are computed between $X$ and $\bar{\sigma}$.)

fluctuations on $\bar{\sigma}$ would be minimal while $\vec{X}$ would be similarly affected. The opposite case, $\tau_{I} \rightarrow \infty$, would produce reverse effects with much higher noise levels for $\bar{\sigma}$ which would similarly influence $X$. Thus,

$$
\tau_{I}<<\tau_{c} \quad \Longrightarrow \quad \text { noise decreases }
$$

which is the case of application for the averaging principle theory.

In Table 5.1 we present numerical evidence of the remarks above by summarizing corresponding results from our Monte Carlo simulations. Note in Table 5.1 that we observe the highest noise levels for decreasing $\tau_{c}$ values as we expected.

TABLE 5.1. Numerical effect on noise due to $\tau_{c}$ and $\beta J_{0}$. Note that the standard deviation is divided by at least 2 every time $\tau_{c}$ increases. Results taken from one of several examples (not shown here) displaying a saddle bifurcation behavior.

\begin{tabular}{|c||c|c|c|}
\multicolumn{4}{c}{ Standard Deviation for $\mathrm{X}$} \\
\hline$\tau_{c}$ & $\beta J_{0}=.01$ & $\beta J_{0}=2$ & $\beta J_{0}=-2$ \\
\hline \hline .1 & .05 & .03 & .02 \\
1 & .02 & .01 & .006 \\
5 & .01 & .004 & .003 \\
\hline
\end{tabular}

\section{Conclusions}

In the present work we have (a) developed mathematical prototype hybrid models coupling stochastic and deterministic systems, (b) derived deterministic mesoscopic models from the hybrid in various asymptotic limits and (c) evaluated the extent 

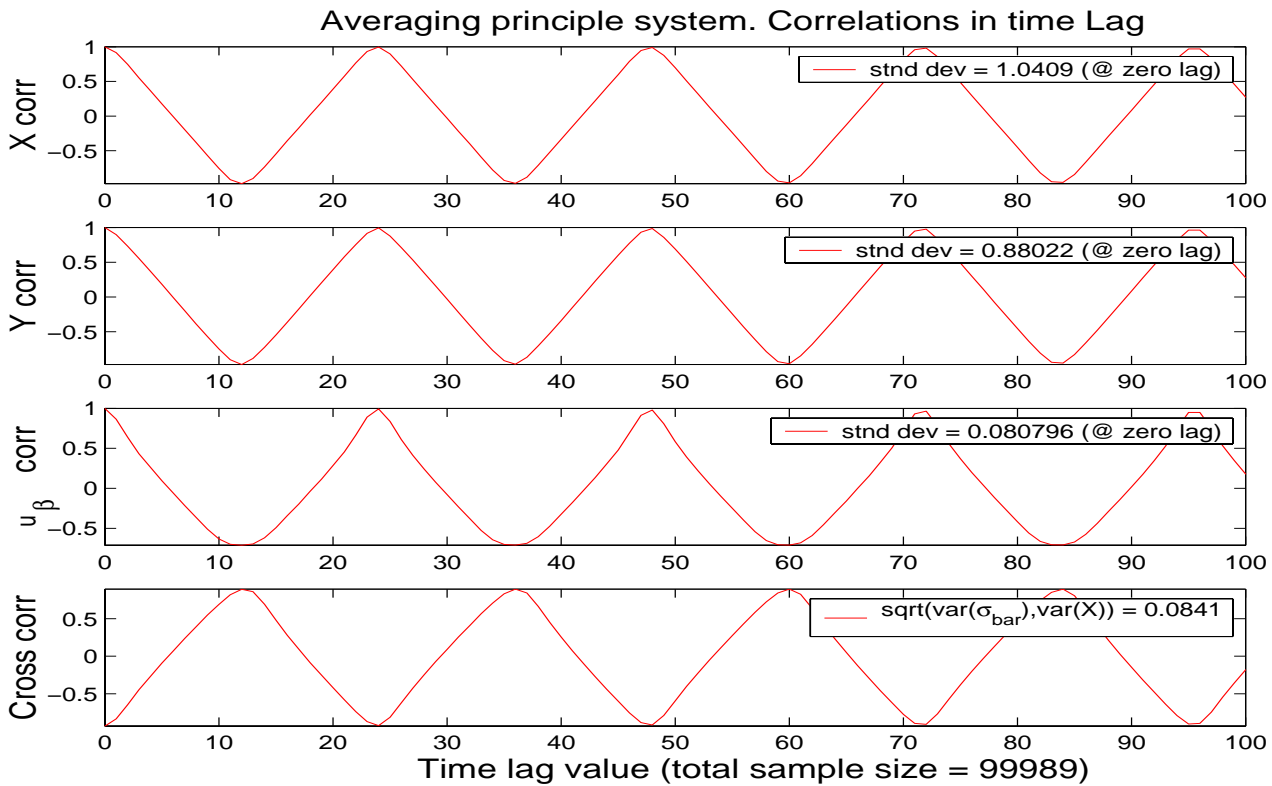

FIG. 4.25. Hopf ODE case: Averaging principle correlations in time lag. Although (4.7) is deterministic we can calculate the correlations corresponding to the coupled system (4.6) as another way of crudely comparing the results between the two systems (compare with Figure 4.24). Parameters: $\tau_{c}=.1$ and $\beta J_{0}=2$. (Cross correlations are computed between $X$ and $\bar{\sigma}$.)

of validity of theoretical predictions by extensive direct numerical simulations and comparisons.

We are convinced, based on all examples presented in this work (and several others not reported here) that in general the average principle predictions and the Monte Carlo simulations completely agree for the parameter regimes for which this theory holds (i.e. faster stochastic $\tau_{I}<<\tau_{c} \rightarrow \infty$ ). We also point out that in general in most applications involving hybrid models the assumption of $\tau_{c} \rightarrow \infty$ is replaced by a finite value of $\tau_{c}$. In this case mean-field theory and in particular the averaging principle could easily lead to false results as we have seen here with our examples.

There is a number of additional issues that are not addressed here which we pursue further in a follow-up work [8]:

- Treatment of systems which include microscopic dynamics displaying phase transitions. In this case we expect significant stochastic fluctuations and agreement between the coupled and the averaged system only for $\tau_{I}<<1$.

- Systematic derivations of stochastic mesoscopic models by employing coarse grained Monte Carlo models [8]. We believe that implementation of the coarse grained Monte Carlo systems will in fact be able to not only include the results of the mean field equations (3.13) but also reproduce the stochastic noise which as we have seen here is essential.

In summary we observe in [8] that the treatment of noise through Coarse Grained Monte Carlo (CGMC) closures correctly represents the coupled system behavior for all cases of relaxation values $\tau$. The behavior of the coupled microscopic system is predicted through CGMC with the advantage of doing so in a fraction of computational time. Furthermore the success of the method is also validated for the phase transition 
regime where metastability and hysteresis phenomena take effect thus validating the CGMC closure presented for an even wider context encompassing complex stochastic dynamics.

Acknowledgments. The research of M.A.K. is partially supported by NSFDMS-0100872, and NSF-ITR-0219211, the research of A.J.M. is partially supported by ONR N00014-96-1-0043, NSF-DMS-9972865 and NSF-CMG-0222133. The research of A.S. is partially supported by ARO-DAAD19-01-10810. M.A.K. and A.J.M. would like to thank the Institute for Pure and Applied Mathematics at the University of California, Los Angeles, where part of this work was carried out during visits in July and August 2003.

\section{REFERENCES}

[1] R.S. Aranson and L. Kramer, The world of the complex ginzburg-landau equation, Reviews of Modern Physics, 74:99-143, 2002.

[2] R.B. Bird, W.E. Stewart, and E.N. Lightfoot, Transport phenomena, Wiley, 1960.

[3] A. Bortz, M. Kalos, and J. Lebowitz, A new algorithm for monte carlo simulation of ising spin systems, J. Comput. Phys., 17:10-18, 1975.

[4] F. Comets, Nucleation for a long range magnetic model, Ann. Inst. H. PoincarProbab. Statist, 23(2):135-178, 1987.

[5] R. Ellis, Entropy, Large Deviations, and Statistical Mechanics, Springer-Verlag, 1985.

[6] K.A. Emanuel and D.J. Raymond, The representation of cumulus convection in numerical models, Meteorological Monographs, Am. Meteorol. Soc., 48, 1993.

[7] M.I. Friedlin and A.D. Wentzell, Random Perturbations of Dynamical Systems, Springer, 1998.

[8] M.A. Katsoulakis, A.J. Majda, and A. Sopasakis, Multiscale couplings in prototype hybrid deterministic/stochastic systems: Part ii, phase transitions and stochastic closures, in progress.

[9] M.A. Katsoulakis, A.J. Majda, and D.G. Vlachos, Coarse-grained stochastic processes and monte carlo simulations in lattice systems, J. Comp. Phys., 186:250-278, 2003.

[10] M.A. Katsoulakis, A.J. Majda, and D.G. Vlachos, Coarse-grained stochastic processes for lattice systems, Proc. Natl. Acad. Sci., 100:782-787, 2003.

[11] M.A. Katsoulakis and D.G. Vlachos, Hierarchical kinetic monte carlo simulations for diffusion of interacting molecules, J. Chem. Phys., 112(18), 2003.

[12] I.G. Kevrekidis, L.D. Schmidt, and R. Aris, Surf. Sci., 137:151, 1984.

[13] B. Khouider, A.J. Majda, and M.A. Katsoulakis, Coarse-grained stochastic models for tropical convection and climate, Proc. Natl. Acad. Sci., 100:11941-11946, 2003.

[14] C. Kipnis and C. Landim, Scaling Limits of Interacting Particle Systems, Springer, 1999.

[15] D.A. Lavis and G.M. Bell, Statistical Mechanics of Lattice Systems 1: Closed-Form and Exact Solutions, Springer, 1999.

[16] C.D. Levermore and M. Marcel, The complex ginzburg-landau equation as a model problem, Lectures in Appl. Math., 31:141-190, 1996.

[17] T.M. Liggett, Stochastic Interacting Systems: Contact process, Voter and Exclusion Process, Springer-Verlag, Berlin Heidelberg, 1999.

[18] A.J. Majda and B. Khouider, Stochastic and mesoscopic models for tropical convection, Proc. Nat. Acad. Sci., 99:1123, 2002.

[19] A.J. Majda and M.G. Shefter, Models for stratiform instability and convectively coupled waves, J. Atmos. Sci., 58(12):1567-1584, 2001.

[20] A.J. Majda, I. Timofeyev, and E. Vanden Eijnden, A mathematical framework for stochastic climate models, Comm. Pure Appl. Math., 54:891, 2001.

[21] A. De Masi, P.A. Ferrari, and J.L. Lebowitz, Reaction-diffusion equations for interacting particle systems, J. Stat. Phys., 44:589-644, 1986.

[22] A. De Masi and E. Presutti, Mathematical methods for hydrodynamical limits, Lecture Notes in Mathematics, 1501, 1991.

[23] O. Penrose, A mean-field equation of motion for the dynamic ising model, J. Stat. Phys., 63(5/6):975-986, 1991. 
[24] D.G. Vlachos, L.D. Schmidt, and R. Aris, The effects of phase transitions, surface diffusion and defects on surface catalyzed reactions: Fluctuations and oscillations, J. Chem. Phys., 93:8306, 1990.

[25] R.M. Ziff, E. Gulari, and Y. Barshad, Kinetic phase transitions in an irreversible surfacereaction model, Phys. Rev. Lett., 56:2553, 1986. 